\title{
Single-cell transcriptome landscape of zebrafish liver reveals hepatocytes and immune cell interactions in understanding nonalcoholic fatty liver disease
}

\author{
Yingyi Huang ${ }^{1,2,10}$, Xiang Liu ${ }^{1,2,10}$, Hong-Yan Wang ${ }^{1,3}$, Jian-Yang Chen ${ }^{4,5}$, Xianghui Zhang, \\ Yubang Li ${ }^{1,7}$, Yifang Lu ${ }^{1,8}$, Zhongdian Dong ${ }^{9}$, Kaiqiang Liu ${ }^{1,3}$, Zhongduo Wang ${ }^{9}$, Qian Wang ${ }^{1,3}$, \\ Guangyi Fan ${ }^{4,5}$, Jun Zou ${ }^{2}$, Shanshan Liu ${ }^{4,5}$, Changwei Shao ${ }^{1,3} *$ \\ ${ }^{1}$ Key Lab of Sustainable Development of Marine Fisheries, Ministry of Agriculture and Rural Affairs, Yellow Sea \\ Fisheries Research Institute, Chinese Academy of Fishery Sciences, 266072 Qingdao, Shandong, China \\ ${ }^{2}$ College of Fisheries and Life Science, Shanghai Ocean University, 201306 Shanghai, China \\ ${ }^{3}$ Laboratory for Marine Fisheries Science and Food Production Processes, Qingdao National Laboratory for \\ Marine Science and Technology, 266071 Qingdao, China \\ ${ }^{4}$ BGI-Qingdao, BGI-Shenzhen, 266555 Qingdao, China \\ ${ }^{5}$ Qingdao-Europe Advanced Institute for Life Sciences, BGI-Shenzhen, 266555 Qingdao, China \\ ${ }^{6}$ College of Marine Technology and Environment, Dalian Ocean University, 116023 Dalian, Liaoning, China \\ ${ }^{7}$ College of Life Science, Shandong Normal University, 250000 JiNan, Shandong, China \\ ${ }^{8}$ School of Marine Sciences, Ningbo University, 315211 Ningbo, Zhejiang, China \\ ${ }^{9}$ Key Laboratory of Aquaculture in South China Sea for Aquatic Economic Animal of Guangdong Higher \\ Education Institutes, Fisheries College, Guangdong Ocean University, Zhanjiang 524025, China \\ ${ }^{10}$ These authors contributed equally \\ *Correspondence: liushanshan@genomics.cn; shaocw@ysfri.ac.cn
}




\begin{abstract}
Zebrafish have emerged as an attractive animal model for studying nonalcoholic fatty liver disease (NAFLD). However, little is known about the cell types and intercellular interactions in zebrafish liver. Here, we established a liver atlas that consists of 10 cell types using single-cell RNA sequencing. By examining the heterogeneity of hepatocytes and analyzing the expression of NAFLD-associated genes in the specific cluster, we provide a potential target cell model to study NAFLD. Additionally, our analysis identified two distinct resident macrophages with inflammatory and noninflammatory functions and characterized the successive stepwise development of $\mathrm{T}$ cell subtypes in the liver. Importantly, we uncovered possible molecular mechanisms and revealed the central regulation of macrophages on target cells of fatty liver by analyzing the cellular interaction between hepatocytes and immune cells. Our data provide valuable information for future research on NAFLD in zebrafish.
\end{abstract}

Keywords: scRNA-seq; zebrafish; NAFLD; hepatocytes; immune cells

\title{
Introduction
}

The liver is a vital organ in the body of vertebrates that exerts essential metabolism and immune function (1). Liver dysfunction leads to a series of diseases, including viral hepatitis, tumors, fibrosis, cirrhosis and fatty liver (2). Among them, nonalcoholic fatty liver disease (NAFLD) has become a common cause of chronic liver disease worldwide, accounting for $24 \%$ of liver diseases (3). Typical NAFLDs include nonalcoholic fatty liver (NAFL), nonalcoholic steatohepatitis (NASH), liver fibrosis, liver cirrhosis and NAFLD-related liver cancer. The progression of NAFLD to liver fibrosis and cirrhosis greatly increases the mortality of liver-related diseases. However, the pathogenesis of NAFLD has yet to be fully elucidated due to the complexity of mechanisms and the ethical issues involved in human experiments. Thus, the establishment of a NAFLD animal model in which the mechanism of liver function has been relatively well studied is of great clinical importance. 
Currently, the "multiple hit" hypothesis has been widely accepted to explain the complexity of NAFLD (4). This concept involves insulin receptor (IR), lipotoxicity, inflammatory response, genetic polymorphism and epigenetics, adipokines and liver factors, bile acid (BA), and gut microbiota (GM) (5). The accumulation of lipid toxic substances caused by the impairment of hepatocyte lipid metabolism is the key to the occurrence of nonalcoholic steatohepatitis (NASH) (6). Other factors, such as dysfunction of cytokines and adipokines, ATP deficiency, uric acid toxicity and products of intestinal microorganisms, may also be involved in regulating the development of lipotoxic stress, injury and inflammation of hepatocytes (7).

Among the many factors affecting the occurrence and development of NAFLD, the important role of immune cell activation should not be ignored (8). Abnormalities in the liver systemic immune response were found to be important factors in the progression of NAFL to NASH and liver fibrosis (9). Macrophages are involved in the pathogenesis of NAFLD (10-12). In terms of cellular functions, liver macrophages can be divided into M1 (inflammatory) and M2 (noninflammatory) macrophages. M1 macrophages promote the production of inflammatory cytokines and reactive oxygen species and are involved in the progression of NAFLD liver inflammation. In contrast, M2 macrophages execute immune regulation, inhibit parasitic infection and strengthen tissue remodeling and are involved in the progression of NAFLD liver fibrosis (13-15). Macrophage-associated factors such as pattern recognition receptors, complement molecules and STING are suggested to promote the development of NAFLD. In addition, $\mathrm{T}$ cells are critical in maintaining liver immune homeostasis and restricting the progression of liver diseases (16). $\mathrm{CD} 4{ }^{+} \mathrm{CD} 25^{+} \mathrm{FOXP}^{+} \mathrm{T}$ cells (regulatory $\mathrm{T}$ cells, Tregs) play an important role in blocking the transformation of NAFL into NASH and mediating the remission of NASH inflammation and fibrosis (17-19). Additionally, excessive production of Th1 cell-derived proinflammatory cytokines may promote hepatic insulin resistance and NASH (8). A recent study has shown that $\mathrm{CD}^{+} \mathrm{T}$ cells undergo autoaggression through a series of continuous activation steps to mediate the occurrence of liver cancer in NASH patients $(20,21)$. 
Zebrafish (Danio rerio) has become an important model to study liver development and diseases and understand the pathogenesis of major liver diseases. Zebrafish and mammals are similar in hepatocyte composition, function and genetics. However, our understanding of hepatocyte biology in zebrafish models has largely come from knowledge of hepatocytes in juvenile fish obtained using image tracking approaches when the adaptive immune system is not well developed (22), which limits the in-depth study of liver fibrosis and liver cirrhosis. To date, the landscape of human and mouse liver cells has been explored at single-cell resolution, providing valuable information to elucidate liver functions and diseases (23-26). The single-cell atlas of adult zebrafish liver is of great significance to complement mammalian studies to uncover the pathogenesis of liver diseases from evolutionary perspectives.

In this study, we used high-throughput scRNA-seq to undertake an unbiased examination of the cellular landscape of healthy zebrafish liver. We identified 10 cell types using known lineage marker genes and potentially targeted cell models for hepatocytes by analyzing cell subclusters. Our analysis revealed remarkably heterogeneous transcriptomic signatures for the pro- and noninflammatory macrophage subtypes and that associated with the development of $\mathrm{T}$ cell subtypes. Importantly, comparative analysis of unbiased cell interactions between zebrafish and human orthologous genes uncovered possible molecular mechanisms of the interactions between immune cells and hepatocytes in zebrafish liver and the roles of macrophages in regulating target cells of fatty liver. Our data provide valuable information for future investigation on NAFLD in zebrafish and enhance our understanding of the molecular events and cellular mechanisms related to liver functions and diseases.

\section{Results}

\section{Single-cell atlas of zebrafish livers}

To investigate the diversity of zebrafish liver cell types, zebrafish liver tissue cells were prepared for preparation of single-cell libraries using the MGI2000 platform (Figure 1A). Six single-cell libraries were sequenced at a median of 78,178 reads per 
cell, which almost reached saturation, as indicated by read down sampling analysis. Subsequently, after quality filtering, a total of 4268 cells were retained, with a median of 4941 transcripts generated per cell. The Seurat package was used for single-cell data analysis and batch effect removal. Cells were further filtered to reduce the dimensionality of the cells, and 10 clusters were obtained (Figure 1B and 1C). Comparing the proportions of different samples in the same cluster, we found that in each independent sample, the proportion of cells in the same cluster was similar, indicating that our data were unaffected by batch effects and could be used to interpret biological significance (Figure 1D).

Based on T-distributed stochastic neighbor embedding (t-SNE) analysis and differentially expressed gene analysis, we annotated three nonimmune populations and seven immune cell populations. Nonimmune cell types primarily consisted of three hepatocyte subclusters: Hepatocyte (Hep) 1 expressing aqp12 (27) and itih2 (28), Hep 2 expressing ahsg1 (29), apoala (30) and apoa4b.1 (31), and Hep3 expressing msmol (23) and hmgcsl (23). The immune cell types identified included lymphocytes (expressing zap70 (32), il7r (33) and ifngl (34)), neutrophils/monocytes (expressing mmp13a (35), mmp9 (36), lyz (37) and lect2l (38)), and eosinosphils (expressing viml (39), hp (39), cd81a (39) and gata2a (39)). Marco (40), cxcl8a (41) and csflra (42) were highly expressed in noninflammatory macrophages, while grnl (43), mpeg1.1 (44) and $c x c r 4 b$ (45) were predominantly expressed in inflammatory macrophages. Additionally, pax5 (46) and $c d 79 a$ (47) exhibited peak expression specifically in B cells, while rhag (48) and transcripts of prdx2 (49) and slc4ala (50) were found only in erythrocytes (Figure 1E). Our data indicate that the cell types in zebrafish liver are conserved with those in the liver of mammals. Therefore, with the advantage of the similarity of the cell types and marker genes, we suggest that we can perform real-time tracking of their morphology and behaviors while modeling various liver diseases by marking individual liver cell types. 


\section{Identification of hepatocyte subtypes in zebrafish}

Previous studies have shown that the composition and function of hepatocytes in zebrafish are similar to those in mammals (51). To determine potential cell types involved in the pathogenesis of liver diseases, we performed reclustering analysis on hepatocytes and identified six distinct subclusters (Figure 2A). Our results showed that cluster 2 was the predominant type of hepatocyte (Figure 2B). To determine the characteristics of subclusters, we examined the cell proportion and DEGs in each cluster, revealing that the numbers of genes expressed were highly variable. For example, cela1.5 (52) and cela1.2 (52) were mainly expressed in cluster 0, while $f g g$ (53), cfhl4 and apom (54) exhibited peak expression in cluster 2. Moreover, pou2f3 (55) and fabplb.1 (56) were highly expressed in cluster 4 and cluster 5, respectively (Figure 2B). The expression profiles of DEGs showed significant heterogeneity of cell subclusters (Figure 2C). To systematically investigate the features of distinct hepatocyte subtypes, we performed GO analysis for the DEGs of each cluster (Figure 2C). Our data showed that genes expressed in cluster 1 were enriched in "Oxidative phosphorylation", "Proteasome" and "Cholesterol Biosynthesis", and those expressed in cluster 2 were enriched in "Ribosomal Proteins", "Response to estrogen" and "Hemostasis". Additionally, "immune response", "NOD-like receptor signaling pathway" and "apoptosis" were noted in cluster 3, whereas "protein processing in endoplasmic reticulum" and "cellular response to hypoxia" were noted in cluster 4. Notably, cluster 5 was enriched with genes related to "Alcohol metabolic process", "PPAR Signaling Pathway" and "Metabolism of lipids". Collectively, these results suggest that subpopulations of hepatocytes may play distinct roles in liver homeostasis in zebrafish. In particular, cluster 5 may be vital for lipid metabolism and synthesis. Furthermore, genes related to fatty liver diseases, such as fabplb.1, adcal (57), fabp2 (56), apoala (58), cd36 (59) and acoxl (60), were highly expressed in cluster 5 (Figure 2D), suggesting that cluster 5 may also be involved in the formation of fatty liver. 


\section{The comparison of inflammatory and noninflammatory macrophages}

Hepatic macrophages are key components of the liver immune system and comprise two distinct populations that possess pro- or anti-inflammatory functions (61). Our data showed that these two macrophage populations are present in the zebrafish liver (Figure 3A). We performed comparative analysis on the cell proportion of the two macrophage subclusters and found that there were no apparent differences (Figure 3B). Next, we performed pairwise comparisons using gene set enrichment analysis (GSEA). The functional pathways that were highly represented in the non-inflammatory macrophage population were associated with immunological tolerance, including "complement activation", "oxidative phosphorylation”, "electron transport activity", "fatty acid binding" and "cell-cell recognition" (Figure 3C). In contrast, the functional pathways that were highly represented in the inflammatory macrophage population were related to the inflammatory response, including the "INF- $\gamma$ mediated signaling pathway" and "toll like receptor signaling pathway" (Figure 3D). Furthermore, genes related to the inflammatory response, such as ciita (62), hla-drbl (63) and tlrl (64), were upregulated in inflammatory macs. To understand the underlying molecular mechanisms driving the differentiation of macrophage subclusters, we performed single-cell regulatory network inference and clustering (SCENIC). This analysis predicted the expression networks of transcription factors that were upregulated in pro- or anti-inflammatory macrophages (Figure 3E). For instance, regulons driven by members of the IRF family were enriched in inflammatory macrophages, such as irfl, which are essential in the development and activation of inflammatory macrophages (65), whereas KLF family members were enriched in non-inflammatory macrophages, such as klf3, which play an important role in the inhibition of inflammation (66).

\section{Heterogeneity of $\mathbf{T}$ cell subclusters}

The liver harbors a number of $\mathrm{T}$ cell populations that exert protective and pathogenic functions in infectious, inflammatory and neoplastic liver diseases (16). To gain a comprehensive view of $\mathrm{CD} 4+$ and $\mathrm{CD} 8^{+} \mathrm{T}$ cell heterogeneity in the zebrafish liver, we identified $\mathrm{CD}^{+}$and $\mathrm{CD}^{+} \mathrm{T}$ cells and further performed reclustering (Figure 4A). Based on unsupervised clustering, $\mathrm{CD}^{+} \mathrm{T}$ cells could be divided into 6 subclusters. 
C0_CD8-gzmk (gzmk (67) and gzm3.2 (67)), C1_CD8-rorc (ccl20a.3 (68), il22 (69), and rorc (70)), C2_CD8-ccl38.6 (ccl38a.4 (71), ccl38.6 (71), and ifng1r (72)) and C5_CD8-lta (lta (73), thfrsf9a (73), and egr1 (74)) were consistent with a memoryand/or effector-like phenotype, while C3_CD8-mki67 (top2a (75), hmgb2a (76), and mki67 (77)) and C4_CD8-mcm4 (mcm4 (78), pcna (79), and hspdl (76)) expressed markers of exhaustion and the cell cycle (Figure 4B and Figure 4D). The CD4+ T cells were grouped into 4 transcriptionally distinct states. C0_CD4-foxp3a(foxp3a (80) and tnfrsf9b (81)), C1_CD4-cd28(il10 (82), cd28 (83), and ifng1r), and C2_CD4-cebpb(cebpb (84) and eeflda (85)), which were consistent with helperand/or regulatory- like phenotypes, while C3_CD4-mki67 (top2a, mki67, and stmn1a (86)) expressed markers of exhaustion and cell cycle (Figure 4C and Figure 4F). Since $\mathrm{T}$ cells transition to new states in metabolic diseases, monocle 2 was used to reflect a possible path for differentiation of CD8+ $\mathrm{T}$ cell subclusters (C2_CD8-ccl38.6, C1_CD8-rorc, C0_CD8-gzmk, C5_CD8-lta, C4_CD8-mcm4 and C3_CD8-mki67 followed a continuous trajectory) and CD4+ T cell subclusters (C1_CD4-cd28, C2_CD4-cebpb, C0_CD4-foxp3a and C3_CD4-mki67 followed a continuous trajectory) (Figure 4E and Figure 4G). Our analysis of finer $\mathrm{T}$ cell states provides better resolution of cell states and suggests testable paths for differentiation in the zebrafish liver.

\section{Interaction between hepatocytes and immune cells}

NAFLD undergoes complex pathophysiological processes, which involve a variety of factors and intrahepatic cells, including parenchymal cells and nonparenchymal cells. Importantly, these processes cannot be achieved by the actions of a single cell, rather by the interactions of different cells to form a complex cross-linking network and feedback system. However, the interaction between hepatocytes and immune cells in the liver is still unclear. Therefore, we examined potential ligand-receptor interactions between hepatocytes and immune cells to predict intercellular communication based on CellPhone DB v2.0 in normal adult zebrafish. The two macrophage subclusters identified in this study displayed relatively high numbers of interactions, while other 
cell types showed lower numbers of potential interactions (Figure 5A). Notably, we found that communications between hepatocytes and immune cells are bidirectional. Inflammatory macrophages were predicted to interact with Hep CL5 through the ligand/receptor pairs ptn/plxn2a, cxcl11.6/dpp4, lta/ltbr and cxcl8aldpp4 to promote the inflammatory state of hepatocytes, while hepatocytes regulate macrophage functions in host defense by synthesizing mif and copa combined with the expression of $c d 74 a$ on the surface of macrophages. We also detected that ltalltbr and cxcl8aldpp4 were active between non-inflammatory macrophages and Hep CL5 cells. Notably, the expression of $c d h 1$, il34 and fam $3 c$ by Hep CL5 could lead to the interaction with the aEb7 complex, csflra and adgrg3, respectively, which are expressed in non-inflammatory macrophages. These immune factors were previously reported to be associated with cell proliferation, differentiation and migration. In addition, we predicted the interaction between $\mathrm{T}$ cells and hepatocytes. For example, we found that the aLb2 complex/f11r.1 showed high specificity and activity between CD8-gzmk and hepatocytes. Lta produced by CD8-rorc, CD8-ccl38.6 and CD8-lta could also interact with its receptor ltbr in Hep CL5 cells. In contrast, Hep CL5 produced $c c l 19 a$, which binds to its receptor $c c r 7$ on CD4-cebpb and CD8-gzmk to regulate immune and inflammatory processes. Tnfsflolfas, related to the apoptosis pathway, exhibited high activity between Hep CL5 and T cells (including CD4-mki67, CD8-rorc, CD8-mki67 and CD8-lta), suggesting that hepatocytes play an important role in regulating $\mathrm{T}$ cell apoptosis.

\section{Discussion}

Zebrafish serve as an ideal animal model for the study of liver diseases. Large-scale RNA sequencing has commonly been applied to clarify the cellular repertoire in the liver. In our study, we identified 10 cell types using a single-cell sequencing approach, which is in agreement with the liver cell atlas in humans and rodents $(23,26,87-89)$. Our data on the transcriptional profiles at the single-cell level provide novel insights into the cell repertoire in teleost organs and valuable information to explore zebrafish as an animal model to study the pathogenesis of human liver diseases. 
Fatty liver is one of the common pathological symptoms of human liver diseases. It is mainly caused by the decline of liver metabolic capacity for a variety of reasons, resulting in induction of hepatocyte stress, injury and death and increased susceptibility to liver fibrosis, liver cirrhosis and hepatocellular carcinoma (90). Therefore, it is crucial to establish a zebrafish liver model and study the source and destination of fatty acids in hepatocytes to understand the metabolic basis of fatty liver. Notably, we found that fabplb1 was specifically expressed in cluster 5 . Fabplb1 is a homologous gene of human FABP1 and participates in intracellular lipid metabolism, such as fatty acid uptake, transport, lipid synthesis and storage (91) (92). The current study has shown that FABPl plays a key role in the excessive accumulation of liver fat, resulting in lipotoxicity and oxidative stress in the progression of NAFL to NASH (93), indicating that cluster 5 is an important group involved in lipid metabolism in zebrafish liver. In addition, we uncovered that $c d 36$ was also specifically expressed in cluster 5. $C d 36$ is a homologous gene of human CD36, which is a potential biomarker for NAFLD diagnosis and patient stratification (94). Previous studies have shown that the cause of hepatolipotoxicity is the excessive ability of hepatocytes to manage and output fatty acids (FFAs) in the form of triglycerides (TGs). CD36 fatty acid translocase facilitates FFA uptake and drives hepatosteatosis onset to form NASH (94). This suggests that cluster 5 can be used to study the role of $C D 36$ in lipid toxicity in the pathogenesis of NAFLD. Additionally, we also found that pathogenicity genes such as adcal, fabp2, apoala and acoxl were highly expressed in cluster 5 . In conclusion, we believe that cluster 5 could be a potential target cell model in the process of NAFLD study, and our data can provide a basis for studying the process of hepatitis in zebrafish liver.

A key finding of our study is the discovery of two distinct populations of resident macrophages in zebrafish, providing regulatory networks that affect macrophage polarization in zebrafish. Studies in humans have shown that there are two subtypes of human liver macrophages, which segregate into proinflammatory and immunoregulatory phenotypes(23). In our study, mrclb (95), hmoxla (96), marco and $c d 63$ (97) were placed in non-inflammatory macrophages in zebrafish liver, and these 
genes are known marker genes for immunoregulatory macrophages. It has been suggested that zebrafish liver non-inflammatory macrophages are similar to the profiles of $\mathrm{CD} 68^{+} \mathrm{MARCO}^{+}$cells in the human liver and that of resident Kupffer cells $(\mathrm{KCs})$ in the rodent liver. These cells are self-renewing, nonmigratory and immunoregulatory (23). Conversely, the transcriptional profiles of zebrafish inflammatory macrophages resemble those of $\mathrm{CD}^{+} 8^{+} \mathrm{MARCO}^{-}$cells in humans, which express high levels of $c d 74 a(45), c d 74 b(45), c x c r 4 b(45)$ and $c c r 9 a(45)$ and are inflammatory (23). In addition, it is worth noting that the Marco gene is a homologous gene of human MARCO. MARCO plays a critical role in the progression of various diseases, suggesting that the non-inflammatory macrophage cluster displaying high levels of MARCO expression could serve as a candidate cell type for studying the functions of liver macrophages (23) (98). It was previously reported that IRF1 is a transcriptional regulator that is central in activating macrophages by proinflammatory signals such as the interferon- $\gamma(\mathrm{IFN}-\gamma)$-mediated signaling pathway $(65,99)$. Loss of irf family members IRF1 or IRF8 causes severe susceptibility to infections in mammals $(100,101)$. Consistent with these studies, it is not surprising that irfl is highly expressed in M1 phenotype macrophages (inflammatory) (Figure $2 \mathrm{E})$. This suggests that they are the key transcription factors for the differentiation of M0 phenotype macrophages into M1 phenotype macrophages in zebrafish. Inflammation and fibrosis of the liver depend on the balance regulation between two KC subsets, inflammatory macrophages and noninflammatory macrophages, which are also known as M1 and M2 macrophages $(102,103)$. Previous studies revealed that during the development of NAFLD, the ratio of M1/M2 macrophages gradually increased, so the reversal of the anti-inflammatory phenotype was suggested to enhance anti-inflammatory signaling and improve the progression of NAFLD (104) (2). Hence, our study provides a further understanding of the therapeutic strategy of using zebrafish to reverse the phenotype of Kupffer cells.

Our study reveals that diverse $\mathrm{T}$ cells are present in zebrafish liver. We identified four CD4+ $\mathrm{T}$ cell subtypes and six CD8+ $\mathrm{T}$ cell subtypes based on $\mathrm{T}$ cell signature genes, such as foxp3a, cd28, cebpb, mki67, gzmk, rorc, ccl38.6, mcm4 and lta. 
Zebrafish foxp $3 a$, a homolog of human FOXP3, has proven to be a key transcription factor of Treg cells (105). $C D 28$ and $C E B P B$ are involved in T cell proliferation and the differentiation of $\mathrm{T}$ helper CD4+ $\mathrm{T}$ cells, respectively $(106,107)$. A recent study has shown that GZMK+ CD8+ T cells are associated with inflammatory aging and age-related dysfunction of the immune system (108). In this study, we found that $\mathrm{CD} 8+\mathrm{T}$ cells expressed $g z m k$, indicating that GZMK+ CD8+ $\mathrm{T}$ cells are present in the zebrafish liver and may play conserved roles in aging-related immune functions. CCL3 promotes the differentiation of primed CD8+ $\mathrm{T}$ cells into effector cells and coordinates their migration. The ccl38.6 ( $c c l 3$ homolog) gene was expressed in zebrafish CD8+ T cells and could be important in the homing and migration of CD8+ T cells. In addition, we identified CD8-lta, CD8-rorc and exhausted T cells (i.e., CD4-mki67, CD8-mki67 and CD8-mcm4) with high expression of cell cycle-related genes, which is consistent with observations in humans (76). Future investigations are required to determine whether predicted gene markers are expressed by different subtypes of $\mathrm{T}$ cells isolated from zebrafish.

Liver immune cells play an important role in mediating the inflammatory response and fibrosis of hepatocytes in the progression of NAFLD. We analyzed immune-hepatocyte cytokine/receptor pairs and their interaction networks and found that macrophages could be involved in regulating the inflammatory response of hepatocytes. cxcl11.6/dpp4 showed specifically high activity between inflammatory macrophages and hepatocytes. cxcl11.6 is homologous to human $c x c l 9$, which was shown to be upregulated in patients with NAFLD and associated with the promotion of the progression of NAFLD to hepatocellular carcinoma $(109,110)$. It is tempting to speculate that cxcl11.6 may be considered a candidate gene for studying the inflammatory response of inflammatory macrophages and hepatocytes in the context of NAFLD in a zebrafish model. MIF exerts antifibrotic effects in liver fibrosis via $C D 74$, and the $M I F / C D 74$ interaction is considered a target for the treatment of liver diseases (111). Based on this, the establishment of a zebrafish mif knockout model is of great significance to study the progress and treatment strategy of liver fibrosis. 
Our study confirmed the presence of CD74a+ hepatocytes and miflcopa-producing macrophages in zebrafish liver. Neutrophils, $\mathrm{CD}^{+} \mathrm{T}^{\mathrm{T}}$ cells, $\mathrm{CD} 8^{+} \mathrm{T}$ cells, NKT cells and other immune cells are known to be involved in the pathological processes of NAFLD (112). Specific types of T cells, including CD8-rorc, CD8-ccl38.6 and CD8-lta, were found in the zebrafish liver, suggesting that ltalltbr-mediated interaction of $\mathrm{CD}^{+} \mathrm{T}$ cells and hepatocytes could potentially play a role in NAFLD. On the other hand, our results showed that $c c r 19$ secreted by hepatocytes acted on the receptor $c c r 7$ in $\mathrm{CD} 4-c e b p b$ and $\mathrm{CD} 8$-gzmk to mediate $\mathrm{T}$ cell migration, while tnfsf10 secreted by hepatocytes might act on the receptor fas in CD4-mki67, CD8-mki67, CD8-rorc and CD8-lta to mediate the apoptosis of T cells. Our identification of these cytokine/receptor pairs in zebrafish liver provides insights into the application of zebrafish models in research into liver diseases and screening of candidate drug targets.

In summary, this work has established the first comprehensive single-cell transcriptomic atlas of zebrafish liver, which provides new insights into the molecular mechanism of zebrafish liver function. Possible candidate cells related to NAFLD and immune cell subtypes that mediate the liver immune response in zebrafish liver have been identified. Bidirectional communication between hepatocytes and immune cells and ligand/receptor interactions were detected. Our study provides a basis to explore zebrafish as a model to study liver functions and NAFLD.

\section{Material and methods}

\section{Biological samples and the ethical use of animals.}

The experimental procedures using zebrafish were approved by the Animal Care and Use Committee at the Chinese Academy of Fishery Sciences, and all experimental procedures were performed in accordance with the guidelines for the Care and Use of Laboratory Animals at the Chinese Academy of Fishery Sciences. 


\section{Dissociation of liver cells in zebrafish.}

To obtain a high-quality cell suspension, we strictly controlled the preparation time.

First, the ovaries of Chinese tongue sole were dissected on ice and washed twice with DMEM, and other tissues adhered to the ovaries were removed with tweezers. The ovarian tissue was cut with a blade to form the tissue homogenate. The tissue homogenate was transferred to the combined enzyme digestion solution $(0.025 \%$ trypsin + collagenase in $400 \mu \mathrm{L} \mathrm{DMEM})$. After heating in a $30^{\circ} \mathrm{C}$ water bath for $5-10$ min, digestion was rapidly terminated with $6 \sim 7 \mathrm{~mL}$ of DMEM. The digested cell suspension was filtered with a $100-\mu \mathrm{m}$ filter first and filtered again with a $40-\mu \mathrm{m}$ filter to collect approximately $7 \mathrm{~mL}$ of the filtered cell suspension into a $15-\mathrm{mL}$ tube. Centrifugation was performed with a horizontal rotor in a cryogenic centrifuge at 400 $\times \mathrm{g}$ for $5 \mathrm{~min}$. After removing the supernatant, $7 \mathrm{~mL}$ of PBS was added, centrifuged at $400 \times \mathrm{g}$ for $5 \mathrm{~min}$, and washed twice. Finally, 0.05\% BSA in PBS was added, and the cells were resuspended. The cells were counted using a hemocytometer under a microscope at a concentration of 1000 2000 cells per $1 \mu \mathrm{L}$.

\section{ScRNA-seq library preparation and sequencing.}

The scRNA-seq library was constructed immediately after the cell suspension was prepared. We used the DNBelab C Series Single Cell RNA Library Preparation Kit based on droplet microfluidics technology for library construction. In detail, we pooled 110,000 cells per library with an average concentration of 1000 cells/ $\mu \mathrm{L}$ and activity greater than $80 \%$. Cells were prepared as droplets in which cell lysis and mRNA capture were performed using the DNBelab C4 portable single-cell system. Single-cell microdroplets were recovered by the emulsion breaking recovery system, after which magnetic bead-captured mRNA was transcribed into cDNA and subjected to 16 cycles of PCR for cDNA enrichment. Finally, cDNA products were used to prepare single-stranded DNA libraries by various steps, such as shearing, end repair, ligation, 12 cycles of PCR, denaturation, circularization, and digestion. Then, $10 \mathrm{ng}$ of the digested product was obtained for sequencing using the MGISEQ 2000 platform.

\section{ScRNA-seq data preprocessing.}


The raw data obtained by scRNA-seq using the MGISEQ 2000 platform were filtered and demultiplexed using PISA (version 1.10.2) (https://github.com/shiquan/PISA). Reads were aligned to the reference genome from NCBI using STAR (version 2.7.9a) and sorted using sambamba (version 0.7.0). The cell versus gene UMI count matrix was generated using PISA.

\section{Quality control, normalization, removal of doublets and dimensionality reduction.}

For every sample, we used Seurat (https://satijalab.org/seurat/) to obtain ideal cells defined as those expressing the number of genes detected between 200 and 3500, with a mitochondrial percentage of less than 5\%. Using the filtered cells, we performed normalization by the SCTransform function and followed the official website tutorial to remove doublets using the $\mathrm{R}$ package DoubletFinder. Subsequently, for visualization, we performed dimensionality reduction of the data using RunPCA, and the first 50 principal components (PCs) were computed with the 3000 highly variable genes using log-normalized expression values calculated by the SCTransform function. Using the ElbowPlot function, we chose the first 30 PCs for the next steps, including computing the k.param nearest neighbors by the FindNeighbors function, constructing the SNN graph by the FindClusters function, and computing the t-SNE by the RunTSNE function. We saved rds to conduct further downstream integration.

\section{Integration, cell cluster and DE analysis.}

Using the SelectIntegrationFeatures function, we retained the top 3000 scoring features to use when integrating all samples, and we found the anchor features by the FindIntegrationAnchors function, with the normalization method parameter as "SCT". Then, we performed integration by the IntegrateData function. Subsequently, we repeated the above method to recluster the cells. Finally, we performed differential expression (DE) using the FindAllMakers function. 


\section{Gene pathway analysis.}

Gene function enrichment analysis was performed using Metascape (https://metascape.org/gp/index.html).

\section{GSEA Analysis.}

To identify the significantly enriched pathways in the difference of noninflammatory mac and inflammatory mac, we used Gene Set Enrichment Analysis (GSEA, http://software.broadinsitute.org/gsea/index.jsp) to perform enrichment analysis. We first transformed the zebrafish single-cell data matrix into an adult matrix. The 8831 one-to-one homologous genes of humans and zebrafish were obtained by BLAST.

\section{SCENIC Analysis.}

To perform transcription factor network inference, we used the SCEINC R package (version 1.2.4, which corresponds to RcisTarget 1.8.0 and AUCell 1.12.0) to calculate the activity of the regulatory networks on the inflammatory mac and noninflammatory mac datasets using default settings.

\section{Cell-cell communication analysis.}

We extracted CD4+ T cell subclusters, CD8+ T cell subclusters, noninflammatory macrophages, inflammatory macrophages and hepatocyte cluster 5 from the transformed matrix as input and then used the CellPhoneDB Python package to perform cell-cell communication analysis. The algorithm is based on the receptor expression of one cell type and the corresponding ligand expression of another cell type to deduce the enriched receptor ligand interaction between the two cell types. Then, we identified the most relevant cell type-specific interactions between ligands and receptors and only considered the receptors and ligands highly expressed in the corresponding subpopulations. 


\section{Acknowledgements}

This work was supported by the National Key R\&D Program of China (2018YFD0900301); the AoShan Talents Cultivation Program Supported by Qingdao National Laboratory for Marine Science and Technology (2017ASTCP-ES06); the Taishan Scholar Project Fund of Shandong of China to C.S.; the National Ten-Thousands Talents Special Support Program to C.S.; the Central Public-interest Scientific Institution Basal Research Fund, CAFS (No.2020TD19).

\section{Author contributions}

Changwei Shao designed and supervised the research. Xiang Liu, Jian-Yang Chen, and Xianghui Zhang performed all experiments, collected and interpreted the data. Yingyi Huang and Yifang Lu did the bioinformatics analysis of single-cell transcriptomic data. Hong-Yan Wang, Yubang Li, Zhongdian Dong, Kaiqiang Liu, Zhongduo Wang, Qian Wang, Guangyi Fan, Jun Zou, and Shanshan Liu provided essential reagents and suggestions. Xiang Liu and Hong-Yan Wang collected the fish samples. Yingyi Huang and Xiang Liu wrote the manuscript. Yingyi Huang, Xiang Liu and Changwei Shao edited the manuscript and revised it critically. All authors took part in the interpretation of data.

\section{Conflict of interest}

The authors declare that they have no conflict of interest.

\section{Reference}

1. N. Aizarani et al., A human liver cell atlas reveals heterogeneity and epithelial progenitors. Nature 572, 199-204 (2019).

2. D. van der Heide, R. Weiskirchen, R. Bansal, Therapeutic Targeting of Hepatic Macrophages for the Treatment of Liver Diseases. Front Immunol 10, 2852 (2019).

3. Z. M. Younossi et al., Global epidemiology of nonalcoholic fatty liver disease-Meta-analytic assessment of prevalence, incidence, and outcomes. Hepatology 64, 73-84 (2016).

4. Y. L. Fang, H. Chen, C. L. Wang, L. Liang, Pathogenesis of non-alcoholic fatty liver disease in children and adolescence: From "two hit theory" to "multiple hit model". World J Gastroenterol 24, 2974-2983 (2018). 
5. E. Buzzetti, M. Pinzani, E. A. Tsochatzis, The multiple-hit pathogenesis of non-alcoholic fatty liver disease (NAFLD). Metabolism 65, 1038-1048 (2016).

6. B. A. Neuschwander-Tetri, Non-alcoholic fatty liver disease. BMC Med 15, 45 (2017).

7. S. L. Friedman, B. A. Neuschwander-Tetri, M. Rinella, A. J. Sanyal, Mechanisms of NAFLD development and therapeutic strategies. Nat Med 24, 908-922 (2018).

8. J. J. Maher, P. Leon, J. C. Ryan, Beyond insulin resistance: Innate immunity in nonalcoholic steatohepatitis. Hepatology 48, 670-678 (2008).

9. Y. T. Zhan, W. An, Roles of liver innate immune cells in nonalcoholic fatty liver disease. World J Gastroenterol 16, 4652-4660 (2010).

10. G. L. Su, Lipopolysaccharides in liver injury: molecular mechanisms of Kupffer cell activation. Am J Physiol Gastrointest Liver Physiol 283, G256-265 (2002).

11. J. Fan et al., The role of Kupffer cells in non-alcoholic steatohepatitis of rats chronically fed with high-fat diet. Zhonghua Gan Zang Bing Za Zhi 9, 16-18 (2001).

12. Y. Adachi, B. U. Bradford, W. Gao, H. K. Bojes, R. G. Thurman, Inactivation of Kupffer cells prevents early alcohol-induced liver injury. Hepatology 20, 453-460 (1994).

13. M. Bartneck et al., Histidine-rich glycoprotein promotes macrophage activation and inflammation in chronic liver disease. Hepatology 63, 1310-1324 (2016).

14. A. Jindal et al., Fat-laden macrophages modulate lobular inflammation in nonalcoholic steatohepatitis (NASH). Exp Mol Pathol 99, 155-162 (2015).

15. J. Wan et al., M2 Kupffer cells promote M1 Kupffer cell apoptosis: a protective mechanism against alcoholic and nonalcoholic fatty liver disease. Hepatology 59, 130-142 (2014).

16. X. Ficht, M. Iannacone, Immune surveillance of the liver by T cells. Sci Immunol 5, (2020).

17. X. Ma et al., A high-fat diet and regulatory T cells influence susceptibility to endotoxin-induced liver injury. Hepatology 46, 1519-1529 (2007).

18. S. Sakaguchi, T. Yamaguchi, T. Nomura, M. Ono, Regulatory T cells and immune tolerance. Cell 133, 775-787 (2008).

19. C. Ma et al., NAFLD causes selective CD4(+) T lymphocyte loss and promotes hepatocarcinogenesis. Nature 531, 253-257 (2016).

20. D. Pfister et al., NASH limits anti-tumour surveillance in immunotherapy-treated HCC. Nature 592, 450-456 (2021).

21. M. Dudek et al., Auto-aggressive CXCR6(+) CD8 T cells cause liver immune pathology in NASH. Nature 592, 444-449 (2021).

22. B. Novoa, A. Figueras, Zebrafish: model for the study of inflammation and the innate immune response to infectious diseases. Adv Exp Med Biol 946, 253-275 (2012).

23. S. A. MacParland et al., Single cell RNA sequencing of human liver reveals distinct intrahepatic macrophage populations. Nat Commun 9, 4383 (2018).

24. X. Xiong et al., Landscape of Intercellular Crosstalk in Healthy and NASH Liver Revealed by Single-Cell Secretome Gene Analysis. Mol Cell 75, 644-660.e645 (2019).

25. P. Ramachandran, K. P. Matchett, R. Dobie, J. R. Wilson-Kanamori, N. C. Henderson, Single-cell technologies in hepatology: new insights into liver biology and disease pathogenesis. Nat Rev Gastroenterol Hepatol 17, 457-472 (2020).

26. X. Su et al., Single-cell RNA-Seq analysis reveals dynamic trajectories during mouse liver development. BMC Genom 18, 946 (2017). 
27. H. M. Poling, S. K. Mohanty, G. M. Tiao, S. S. Huppert, A comprehensive analysis of aquaporin and secretory related gene expression in neonate and adult cholangiocytes. Gene Expr Patterns 15, 96-103 (2014).

28. X. Kang, L. Bai, X. Qi, J. Wang, Screening and identification of key genes between liver hepatocellular carcinoma (LIHC) and cholangiocarcinoma (CHOL) by bioinformatic analysis. Medicine (Baltimore) 99, e23563 (2020).

29. J. I. Heo et al., Melatonin improves insulin resistance and hepatic steatosis through attenuation of alpha-2-HS-glycoprotein. J Pineal Res 65, e12493 (2018).

30. A. Ieda et al., Ellagic Acid Suppresses ApoB Secretion and Enhances ApoA-1 Secretion from Human Hepatoma Cells, HepG2. Molecules 26, (2021).

31. S. Motoi et al., Serum APOA4 Pharmacodynamically Represents Administered Recombinant Human Hepatocyte Growth Factor (E3112). Int J Mol Sci 22, (2021).

32. J. Li et al., ZAP70 activation is an early event of T cell immunity that involved in the anti-bacterial adaptive immune response of Nile tilapia. Dev Comp Immunol 124, 104177 (2021).

33. K. R. Thomas et al., Activated interleukin-7 receptor signaling drives B-cell acute lymphoblastic leukemia in mice. Leukemia, (2021).

34. S. Jiaxin, W. Shengchen, C. Yirong, W. Shuting, L. Shu, Cadmium exposure induces apoptosis, inflammation and immunosuppression through CYPs activation and antioxidant dysfunction in common carp neutrophils. Fish Shellfish Immunol 99, 284-290 (2020).

35. C. R. Flannery et al., A novel placental tissue biologic, PTP-001, inhibits inflammatory and catabolic responses in vitro and prevents pain and cartilage degeneration in a rat model of osteoarthritis. Osteoarthr Cartil 29, 1203-1212 (2021).

36. S. Lepidi et al., MMP9 production by human monocyte-derived macrophages is decreased on polymerized type I collagen. J Vasc Surg 34, 1111-1118 (2001).

37. D. M. Popescu et al., Decoding human fetal liver haematopoiesis. Nature 574, 365-371 (2019).

38. A. L'Hermitte et al., Lect2 Controls Inflammatory Monocytes to Constrain the Growth and Progression of Hepatocellular Carcinoma. Hepatology 69, 160-178 (2019).

39. C. S. Baron et al., Cell Type Purification by Single-Cell Transcriptome-Trained Sorting. Cell 179, 527-542.e519 (2019).

40. K. K. Acheampong et al., Multiplexed detection of SARS-CoV-2 genomic and subgenomic RNA using in situ hybridization. bioRxiv, (2021).

41. S. Asokan, O. R. Bandapalli, CXCL8 Signaling in the Tumor Microenvironment. Adv Exp Med Biol 1302, 25-39 (2021).

42. G. Ferrero, M. Miserocchi, E. Di Ruggiero, V. Wittamer, A csf1rb mutation uncouples two waves of microglia development in zebrafish. Development 148, (2021).

43. K. Takahashi, S. Nakamura, W. Otsu, M. Shimazawa, H. Hara, Progranulin deficiency in Iba-1(+) myeloid cells exacerbates choroidal neovascularization by perturbation of lysosomal function and abnormal inflammation. J Neuroinflammation 18, 164 (2021).

44. B. R. Moyse, R. J. Richardson, A Population of Injury-Responsive Lymphoid Cells Expresses mpeg1.1 in the Adult Zebrafish Heart. Immunohorizons 4, 464-474 (2020).

45. S. Ye et al., Intravesical CD74 and CXCR4, macrophage migration inhibitory factor (MIF) receptors, mediate bladder pain. PLoS One 16, e0255975 (2021). 
46. E. Melzi, M. S. Rocchi, G. Entrican, M. Caporale, M. Palmarini, Immunophenotyping of Sheep Paraffin-Embedded Peripheral Lymph Nodes. Front Immunol 9, 2892 (2018).

47. N. Marwah et al., Optimal panel of immunohistochemistry for the diagnosis of B-cell non-Hodgkin lymphoma using bone marrow biopsy: a tertiary care center study. Blood Res $\mathbf{5 6}$, 26-30 (2021).

48. R. W. Xia et al., A novel double-variant RHAG allele leads to Rh(mod) phenotype. Transfus Med 29, 460-465 (2019).

49. K. S. Romanello et al., Global analysis of erythroid cells redox status reveals the involvement of Prdx1 and Prdx2 in the severity of beta thalassemia. PLoS One 13, e0208316 (2018).

50. I. Ramasamy, Atypical hereditary spherocytosis phenotype associated with pseudohypokalaemia and a new variant in the band 3 protein. BMJ Case Rep 13, (2020).

51. J. Chu, K. C. Sadler, New school in liver development: lessons from zebrafish. Hepatology 50, 1656-1663 (2009).

52. I. Hachero-Cruzado et al., Characterization of the genomic responses in early Senegalese sole larvae fed diets with different dietary triacylglycerol and total lipids levels. Comp Biochem Physiol Part D Genomics Proteomics 12, 61-73 (2014).

53. C. Espitia Jaimes, R. J. Fish, M. Neerman-Arbez, Local chromatin interactions contribute to expression of the fibrinogen gene cluster. J Thromb Haemost 16, 2070-2082 (2018).

54. Y. Bai et al., ApoM is an important potential protective factor in the pathogenesis of primary liver cancer. J Cancer 12, 4661-4671 (2021).

55. J. Bintz et al., Expression of POU2F3 Transcription Factor Control Inflammation, Immunological Recruitment and Metastasis of Pancreatic Cancer in Mice. Biology (Basel) 9, (2020).

56. I. Graupera et al., Adipocyte Fatty-Acid Binding Protein is Overexpressed in Cirrhosis and Correlates with Clinical Outcomes. Sci Rep 7, 1829 (2017).

57. T. Perli et al., Identification of Oxygen-Independent Pathways for Pyridine Nucleotide and Coenzyme A Synthesis in Anaerobic Fungi by Expression of Candidate Genes in Yeast. mBio 12, e0096721 (2021).

58. E. A. Karavia et al., Apolipoprotein A-I modulates processes associated with diet-induced nonalcoholic fatty liver disease in mice. Mol Med 18, 901-912 (2012).

59. C. G. Wilson et al., Hepatocyte-Specific Disruption of CD36 Attenuates Fatty Liver and Improves Insulin Sensitivity in HFD-Fed Mice. Endocrinology 157, 570-585 (2016).

60. A. He et al., Acetyl-CoA Derived from Hepatic Peroxisomal $\beta$-Oxidation Inhibits Autophagy and Promotes Steatosis via mTORC1 Activation. Mol Cell 79, 30-42.e34 (2020).

61. A. Shwartz, W. Goessling, C. Yin, Macrophages in Zebrafish Models of Liver Diseases. Front Immunol 10, 2840 (2019).

62. M. Buxadé et al., Macrophage-specific MHCII expression is regulated by a remote Ciita enhancer controlled by NFAT5. J Exp Med 215, 2901-2918 (2018).

63. V. van Drongelen et al., HLA-DRB1 allelic epitopes that associate with autoimmune disease risk or protection activate reciprocal macrophage polarization. Sci Rep 11, 2599 (2021).

64. S. Grassin-Delyle et al., The Role of Toll-Like Receptors in the Production of Cytokines by Human Lung Macrophages. J Innate Immun 12, 63-73 (2020). 
65. D. Langlais, L. B. Barreiro, P. Gros, The macrophage IRF8/IRF1 regulome is required for protection against infections and is associated with chronic inflammation. J Exp Med 213, 585-603 (2016).

66. A. J. Knights et al., Krüppel-like factor 3 (KLF3) suppresses NF-кB-driven inflammation in mice. J Biol Chem 295, 6080-6091 (2020).

67. S. M. Salti et al., Granzyme B regulates antiviral CD8+ T cell responses. J Immunol 187, 6301-6309 (2011).

68. A. Y. S. Lee et al., Expression of Membrane-Bound CC Chemokine Ligand 20 on Follicular T Helper Cells in T-B-Cell Conjugates. Front Immunol 8, 1871 (2017).

69. M. St Paul et al., IL6 Induces an IL22(+) CD8(+) T-cell Subset with Potent Antitumor Function. Cancer Immunol Res 8, 321-333 (2020).

70. S. Chellappa et al., CD8+ T Cells That Coexpress ROR $\gamma \mathrm{t}$ and T-bet Are Functionally Impaired and Expand in Patients with Distal Bile Duct Cancer. J Immunol 198, 1729-1739 (2017).

71. M. J. Trifilo, C. C. Bergmann, W. A. Kuziel, T. E. Lane, CC chemokine ligand 3 (CCL3) regulates CD8(+)-T-cell effector function and migration following viral infection. $J$ Virol 77, 4004-4014 (2003).

72. P. Bhat, G. Leggatt, N. Waterhouse, I. H. Frazer, Interferon- $\gamma$ derived from cytotoxic lymphocytes directly enhances their motility and cytotoxicity. Cell Death Dis 8, e2836 (2017).

73. H. H. Workel et al., Transcriptional Activity and Stability of CD39+CD103+CD8+ T Cells in Human High-Grade Endometrial Cancer. Int J Mol Sci 21， (2020).

74. A. Singh, J. Svaren, J. Grayson, M. Suresh, CD8 T cell responses to lymphocytic choriomeningitis virus in early growth response gene 1-deficient mice. J Immunol 173, 3855-3862 (2004).

75. A. Petrelli et al., PD-1+CD8+ T cells are clonally expanding effectors in human chronic inflammation. J Clin Invest 128, 4669-4681 (2018).

76. M. Sade-Feldman et al., Defining T Cell States Associated with Response to Checkpoint Immunotherapy in Melanoma. Cell 176, 404 (2019).

77. P. A. Szabo et al., Single-cell transcriptomics of human T cells reveals tissue and activation signatures in health and disease. Nat Commun 10, 4706 (2019).

78. S. J. Orr et al., Reducing MCM levels in human primary T cells during the G(0)-->G(1) transition causes genomic instability during the first cell cycle. Oncogene 29, 3803-3814 (2010).

79. W. Strzalka, A. Ziemienowicz, Proliferating cell nuclear antigen (PCNA): a key factor in DNA replication and cell cycle regulation. Ann Bot 107, 1127-1140 (2011).

80. X. Li et al., A critical role of foxp3a-positive regulatory T cells in maintaining immune homeostasis in zebrafish testis development. J Genet Genomics 47, 547-561 (2020).

81. J. C. Moore et al., T Cell Immune Deficiency in zap70 Mutant Zebrafish. Mol Cell Biol 36, 2868-2876 (2016).

82. G. Trinchieri, Interleukin-10 production by effector T cells: Th1 cells show self control. J Exp Med 204, 239-243 (2007).

83. C. H. June, J. A. Ledbetter, P. S. Linsley, C. B. Thompson, Role of the CD28 receptor in T-cell activation. Immunol Today 11, 211-216 (1990). 
84. V. J. Lynch, G. May, G. P. Wagner, Regulatory evolution through divergence of a phosphoswitch in the transcription factor CEBPB. Nature 480, 383-386 (2011).

85. S. Zhang et al., Identification of novel isoforms of dairy goat EEF1D and their mRNA expression characterization. Gene 581, 14-20 (2016).

86. C. Zheng et al., Landscape of Infiltrating T Cells in Liver Cancer Revealed by Single-Cell Sequencing. Cell 169, 1342-1356.e1316 (2017).

87. V. L. Payen et al., Single-cell RNA sequencing of human liver reveals hepatic stellate cell heterogeneity. JHEP Rep 3, 100278 (2021).

88. J. Zhao et al., Single-cell RNA sequencing reveals the heterogeneity of liver-resident immune cells in human. Cell Discov 6, 22 (2020).

89. J. Zhao et al., Single-cell RNA sequencing reveals the heterogeneity of liver-resident immune cells in human. Cell Discov 6, 22 (2020).

90. H. Yki-Järvinen, Non-alcoholic fatty liver disease as a cause and a consequence of metabolic syndrome. Lancet Diabetes Endocrinol 2, 901-910 (2014).

91. B. P. Atshaves et al., Liver fatty acid-binding protein and obesity. J Nutr Biochem 21, 1015-1032 (2010).

92. G. Wang, H. L. Bonkovsky, A. de Lemos, F. J. Burczynski, Recent insights into the biological functions of liver fatty acid binding protein 1. J Lipid Res 56, 2238-2247 (2015).

93. T. Mukai, M. Egawa, T. Takeuchi, H. Yamashita, T. Kusudo, Silencing of FABP1 ameliorates hepatic steatosis, inflammation, and oxidative stress in mice with nonalcoholic fatty liver disease. FEBS Open Bio 7, 1009-1016 (2017).

94. P. Rada, Á. González-Rodríguez, C. García-Monzón, M. Valverde Á, Understanding lipotoxicity in NAFLD pathogenesis: is CD36 a key driver? Cell Death Dis 11, 802 (2020).

95. F. Yuan et al., Effects of Des-acyl Ghrelin on Insulin Sensitivity and Macrophage Polarization in Adipose Tissue. J Transl Int Med 9, 84-97 (2021).

96. T. Bise, A. S. de Preux Charles, A. Jaźwińska, Ciliary neurotrophic factor stimulates cardioprotection and the proliferative activity in the adult zebrafish heart. NPJ Regen Med 4, 2 (2019).

97. B. O. Fabriek, C. D. Dijkstra, T. K. van den Berg, The macrophage scavenger receptor CD163. Immunobiology 210, 153-160 (2005).

98. A. M. Georgoudaki et al., Reprogramming Tumor-Associated Macrophages by Antibody Targeting Inhibits Cancer Progression and Metastasis. Cell Rep 15, 2000-2011 (2016).

99. C. A. Jefferies, Regulating IRFs in IFN Driven Disease. Front Immunol 10, 325 (2019).

100. T. Jeyakumar et al., Inactivation of Interferon Regulatory Factor 1 Causes Susceptibility to Colitis-Associated Colorectal Cancer. Sci Rep 9, 18897 (2019).

101. S. Salem, D. Salem, P. Gros, Role of IRF8 in immune cells functions, protection against infections, and susceptibility to inflammatory diseases. Hum Genet 139, 707-721 (2020).

102. V. Maina et al., Bias in macrophage activation pattern influences non-alcoholic steatohepatitis (NASH) in mice. Clin Sci (Lond) 122, 545-553 (2012).

103. L. J. Dixon, M. Barnes, H. Tang, M. T. Pritchard, L. E. Nagy, Kupffer cells in the liver. Compr Physiol 3, 785-797 (2013).

104. F. Tacke, Targeting hepatic macrophages to treat liver diseases. J Hepatol 66, 1300-1312 (2017). 
105. M. Ono, Control of regulatory T-cell differentiation and function by T-cell receptor signalling and Foxp3 transcription factor complexes. Immunology 160, 24-37 (2020).

106. J. H. Esensten, Y. A. Helou, G. Chopra, A. Weiss, J. A. Bluestone, CD28 Costimulation: From Mechanism to Therapy. Immunity 44, 973-988 (2016).

107. J. Blackwell et al., Changes in CEBPB expression in circulating leukocytes following eccentric elbow-flexion exercise. J Physiol Sci 65, 145-150 (2015).

108. D. A. Mogilenko et al., Comprehensive Profiling of an Aging Immune System Reveals Clonal GZMK(+) CD8(+) T Cells as Conserved Hallmark of Inflammaging. Immunity 54, 99-115.e112 (2021).

109. N. Dali-Youcef et al., Interleukin-32 Contributes to Human Nonalcoholic Fatty Liver Disease and Insulin Resistance. Hepatol Commun 3, 1205-1220 (2019).

110. F. Mirshahi et al., Longitudinal studies can identify distinct inflammatory cytokines associated with the inhibition or progression of liver cancer. Liver Int 40, 468-472 (2020).

111. D. Heinrichs et al., Macrophage migration inhibitory factor (MIF) exerts antifibrotic effects in experimental liver fibrosis via CD74. Proc Natl Acad Sci U S A 108, 17444-17449 (2011).

112. R. Loomba, S. L. Friedman, G. I. Shulman, Mechanisms and disease consequences of nonalcoholic fatty liver disease. Cell 184, 2537-2564 (2021). 


\section{Figure legends}

Figure 1 Overview of 10 cell types determined by scRNA sequencing in zebrafish.

A. Schematic representation of the single-cell RNA-seq analysis of zebrafish liver cells. B. Visualization of major testis cell types for 4268 cells in UMAP (unknown is undefined). Different cell types are shown in distinct colors. C. Heatmap showing the expression of the DEGs for the main cell types. Z-scores were calculated by subtracting the average value for the set of data from the value for each cell and dividing by the standard deviation. D. Pie chart showing proportion of 10 cell types. E. Violin plots of the normalized expression of marker genes for 10 cell types.

Figure 2 Analysis of hepatocyte subclusters.

A. UMAP of hepatocytes reclustering. B. Histogram of cell proportion in hepatocyte subclusters. The upper picture represents the cell proportion of hepatocyte subclusters, and the lower picture represents the top 10 DEGs in the clusters. C. Bubble plot and heatmap of hepatocytes in different subclusters. The bubble plot represents the terms of subclusters in hepatocytes, and the heatmap represents the expression of DEGs in subclusters of hepatocytes. D. Feature plot of the key genes of various liver-associated diseases in CL5.

Figure 3 Comparison of differences between noninflammatory macrophages and inflammatory macrophages.

A. Feature plot of the expression of key genes (marco and cd74a) in all cell types. Color scale: purple, high expression; white, low expression. B. Stacked histogram of the proportion of noninflammatory macrophages and inflammatory macrophages in different samples. C. GSEA enrichment plots of GO signaling pathways between noninflammatory macrophages and inflammatory macrophages. D. GSEA enrichment plots of GO signaling pathways and boxplots of key component genes associated with the inflammatory response between noninflammatory macrophages and inflammatory macrophages. E. Heatmap of the inferred transcription factor gene regulatory networks (SCENICs) between noninflammatory macrophages and inflammatory macrophages. Numbers between brackets indicate the (extended) regulons for respective transcription factors. Color scale: red, high expression; blue, low expression.

Figure 4 Analysis of CD8+ $T$ cell subclusters and CD4+ $T$ cell subclusters. A. $\mathrm{t}-\mathrm{SNE}$ plot visualization of CD8+ $\mathrm{T}$ cells and CD4+ $\mathrm{T}$ cells, color coded for the identified T cell phenotypes. (Purple indicates CD8+ T cells, and red indicates CD4+ T cells). B. $t-S N E$ plot visualization of CD8+ T cell subclusters, color coded for the identified CD8 $+\mathrm{T}$ cell phenotypes. C. t-SNE plot visualization of CD4+ T cell subclusters, color coded for the identified CD4+ T cell phenotypes. D. Heatmap showing the expression of the top 20 DEGs for CD8+ T cell subclusters. Z-scores were calculated by subtracting the average value for the set of data from the value for each cell and dividing by the standard deviation. E. Developmental pseudotime trajectory of $\mathrm{CD} 8+\mathrm{T}$ cell subclusters. The upper figure represents the pseudotime process of the different CD8+ T cell subclusters, color coded for the identified CD8+ $\mathrm{T}$ cell phenotypes; the figure below shows the differentiation of different cell subclusters; the darker the color, the lower the degree of differentiation. F. Heatmap 
showing the expression of the top 20 DEGs for CD4+ T cell subclusters. Z-scores were calculated by subtracting the average value for the set of data from the value for each cell and dividing by the standard deviation. G. Developmental pseudotime trajectory of $\mathrm{CD} 4+\mathrm{T}$ cell subclusters. The upper figure represents the pseudotime process of the different CD4+ T cell subclusters, color coded for the identified CD4+ $\mathrm{T}$ cell phenotypes; the figure below shows the differentiation of different cell subclusters; the darker the color is, the lower the degree of differentiation.

Figure 5 Interaction in hepatocytes-immune cells.

A. Heatmap showing the interaction scores between hepatocyte subcluster-5 and immune cells. Color scale: Purplish red, high expression; blue, low expression. B. Overview of selected interactions of receptors and ligands expressed by hepatocyte subcluster-5 and immune cells. the color of the genes: red, receptors; black, ligands. $\mathbf{C}$. Interaction pattern between hepatocytes and immune cells.

Dataset supplement 1 DEGs in all cell types in zebrafish liver.

Dataset supplement 2 DEGs in all cell types in hepatocyte subclusters.

Dataset supplement 3 DEGs in all cell types in CD4+ T cell subclusters.

Dataset supplement 4 DEGs in all cell types in CD8+ $\mathrm{T}$ cell subclusters. 


\section{A}

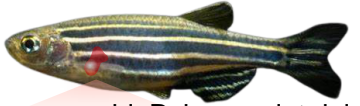

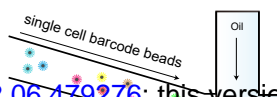

/ $90.1101 / 2022.02 .06 .479276$; this tersion pestedfebryary 9,2022 . The copyright holder for this

$\int \begin{array}{ll}\because & \text { (which } \\ 0 & \cdots \\ 0 & \infty\end{array}$

liver

which was not certhed by peer review, is the authoffunder. Al

B

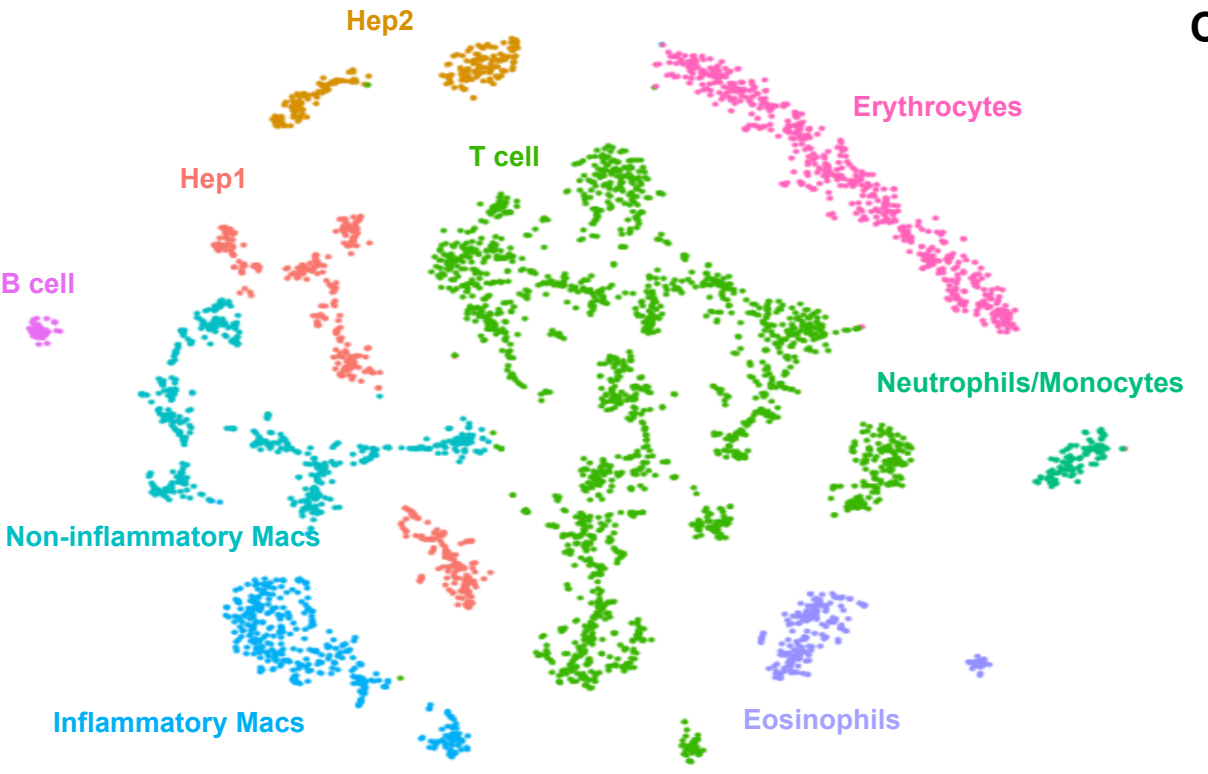

C

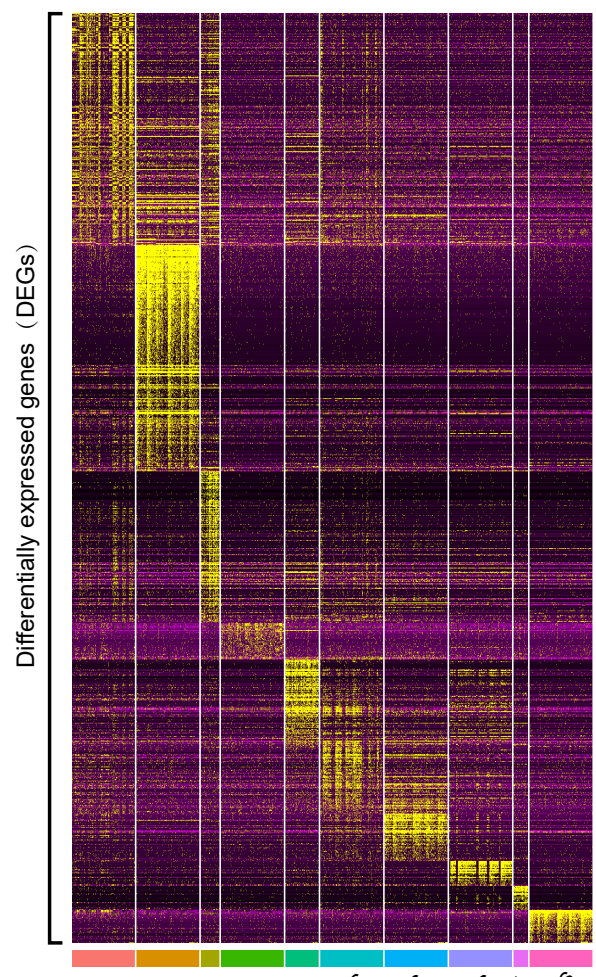

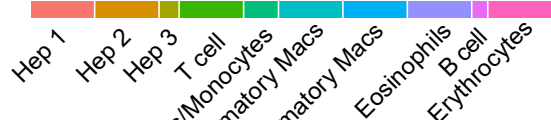

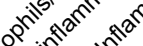

Expression

E

Hep 1

$\square$ Hep 2

$\square$ Hep 3

$\square$ T cell

Neutrophils/Monocytes

- Non-inflammatory Macs

- Inflammatory Macs

$\square$ Eosinophils

$\square$ B cell

$\square$ Erythrocytes
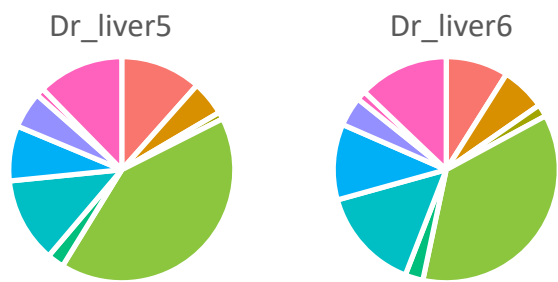


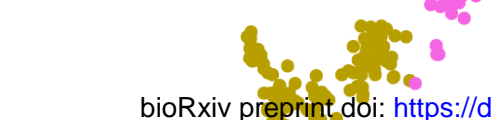

$30 \%$

bioRxiv preprint doi: https://doi.org/10.1101/2022.02.06.479276; this yersion posted February 9, 2022. The copyright holder for this preprint

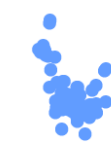

(which was

All rights reserved. No reuse allowed without permission.

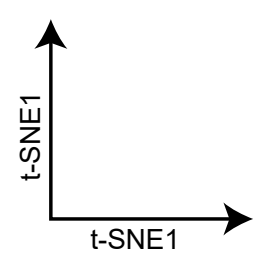

C
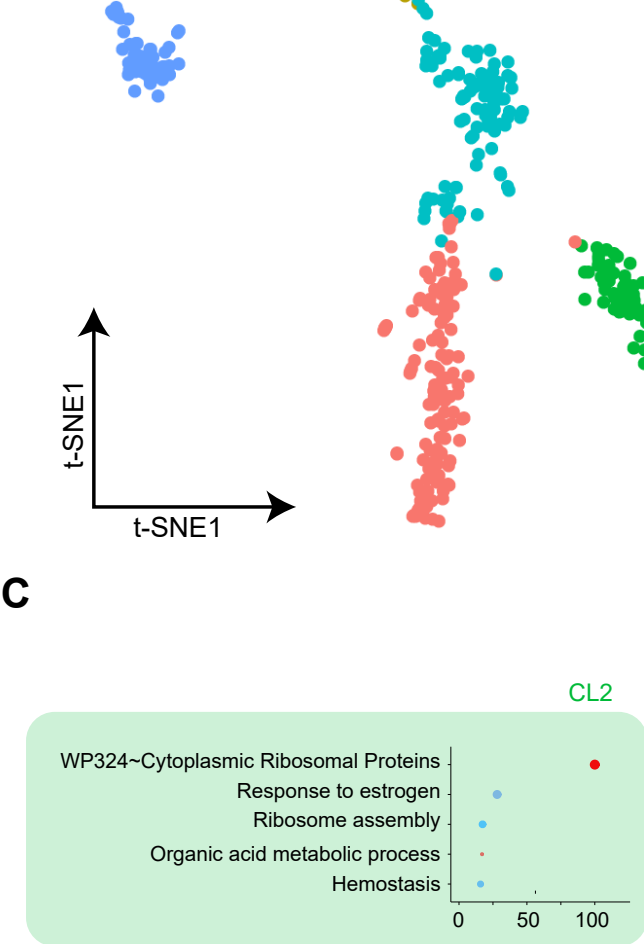

- CLO

- CL1

- CL2

- CL3

- CL4

- CL5

-SNE1

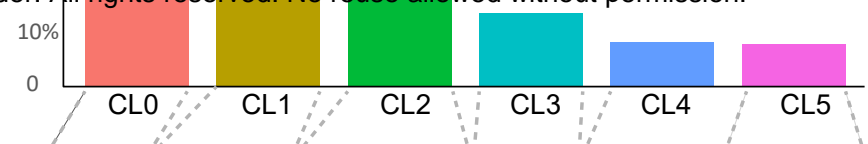

cela1.5 tmem1761.2 fgg

cpa4 cfl11

cela1.2 tspan34

endou plac8.2

cela1.1 Igals2b

pla2g1b phlda2

spink4 s100u

cpb1 itga6l

pglyrp6 eps8/3b

cpa2 ca4b

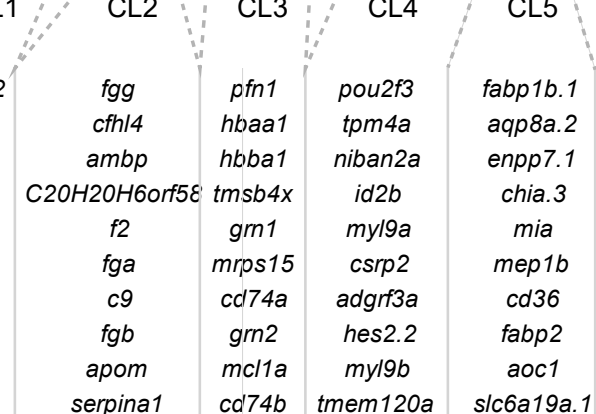

CL3
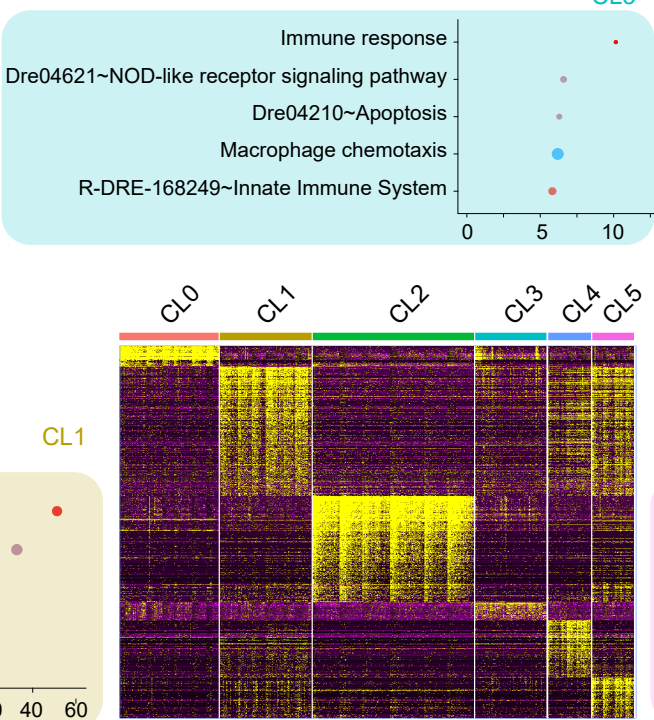

Expression

$\begin{array}{lll}-1 & 0 & 1\end{array}$
Dre04141 Protein processing in endoplasmic reticulum R-DRE-72163 mRNA Splicing - Major Pathway R-DRE-168249 Innate Immune System R-DRE-199991 Membrane Trafficking R-DRE-1234174 Cellular response to hypoxia 20

D

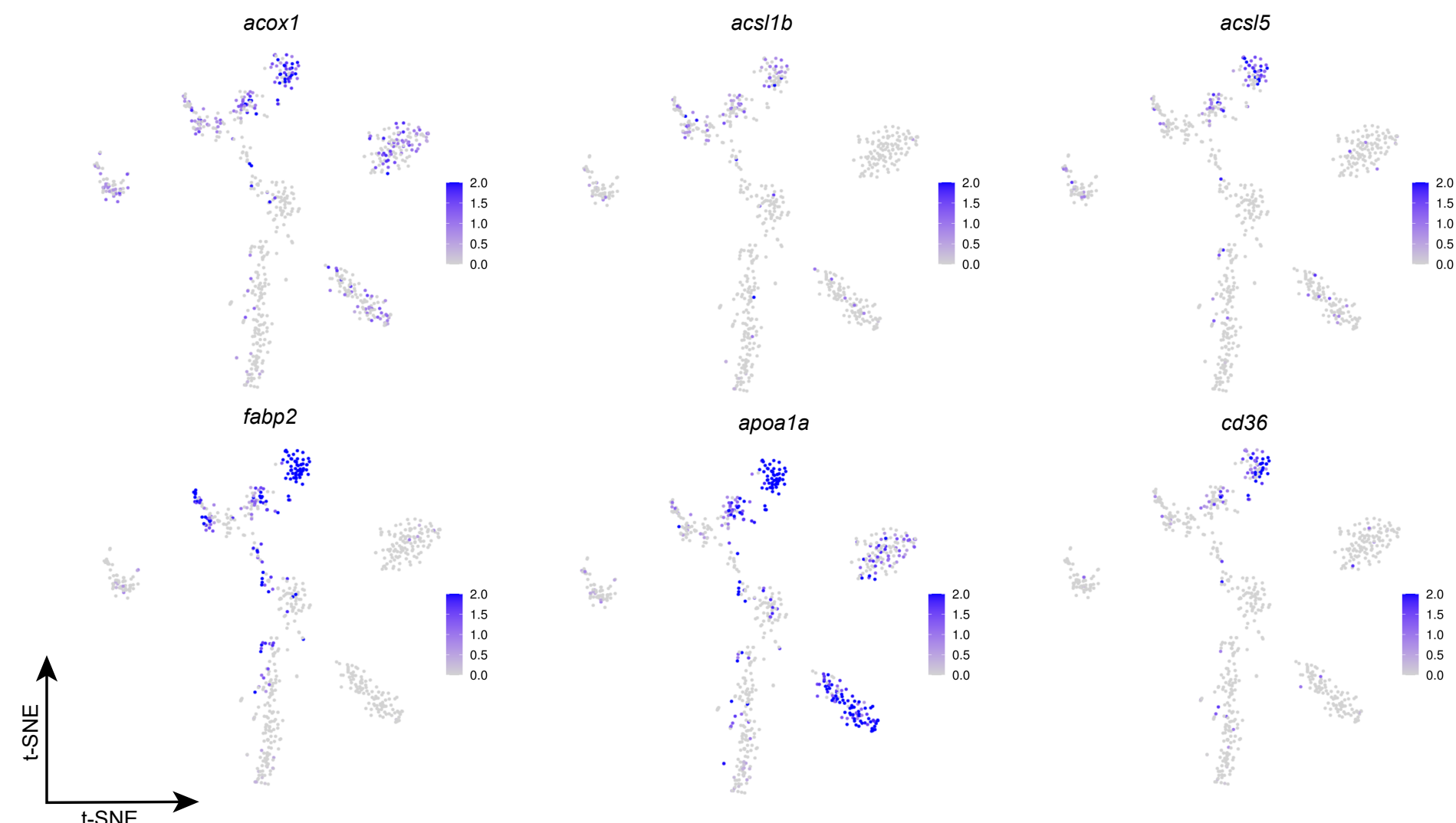



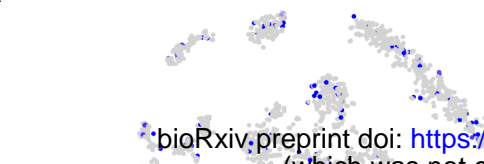

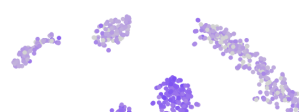

s://doi.org/10.1101/2022.02.06.47927.6 this version pos

$\therefore$ bioRxiv.preprint doi: htps://doi.org/10.1101/2022.02.06.47927.6. this version posted February 9, 2022 The copyright holder for this preprint
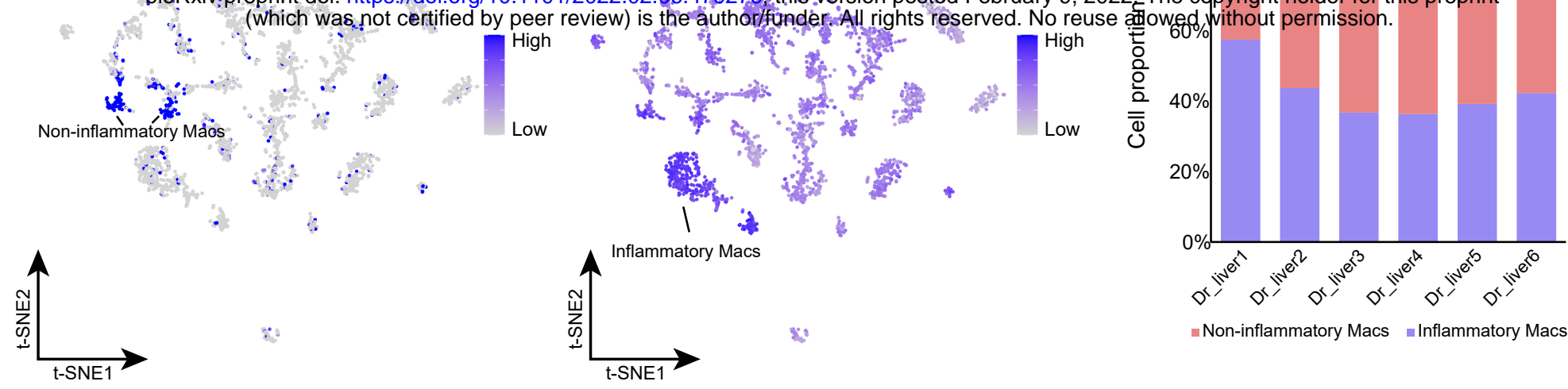

C
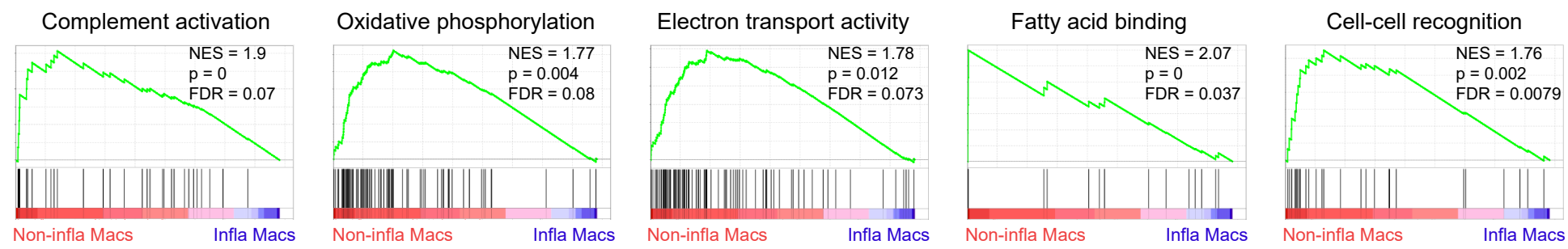

D
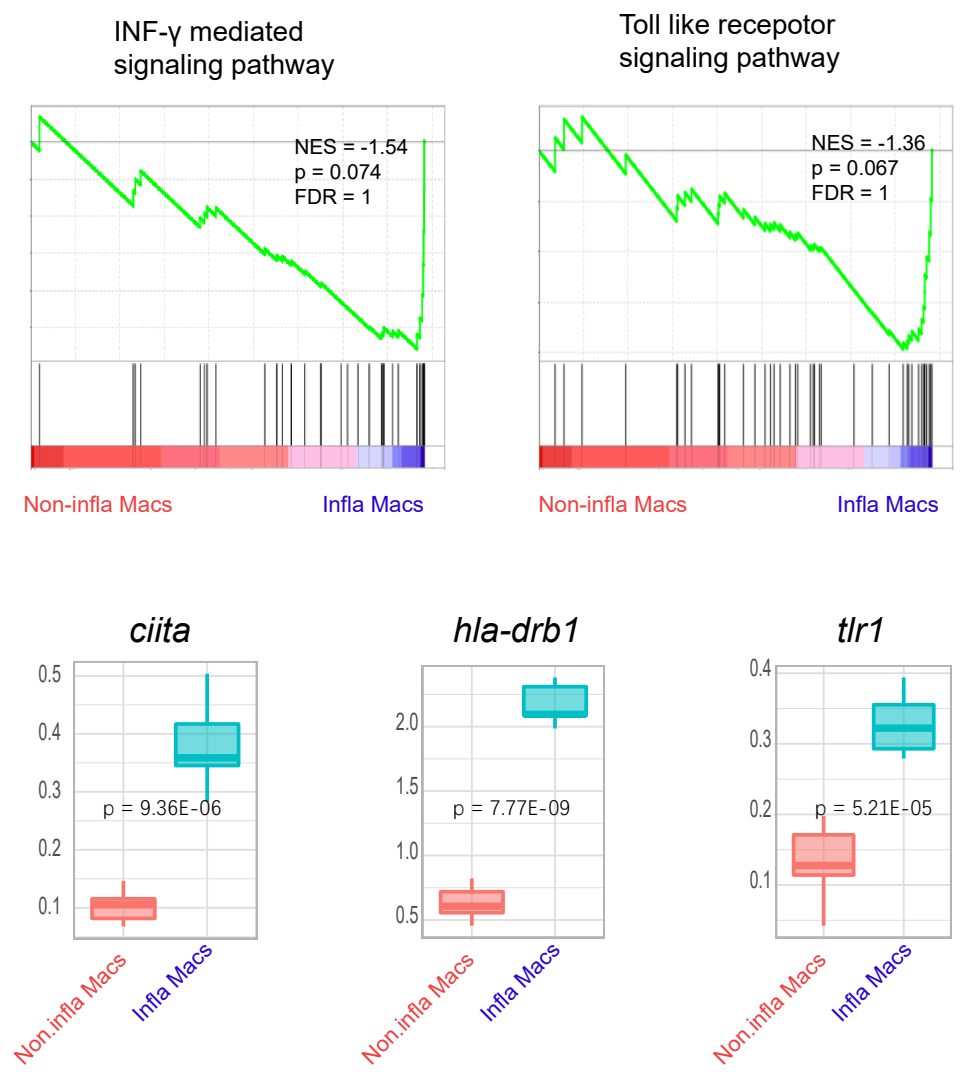

E
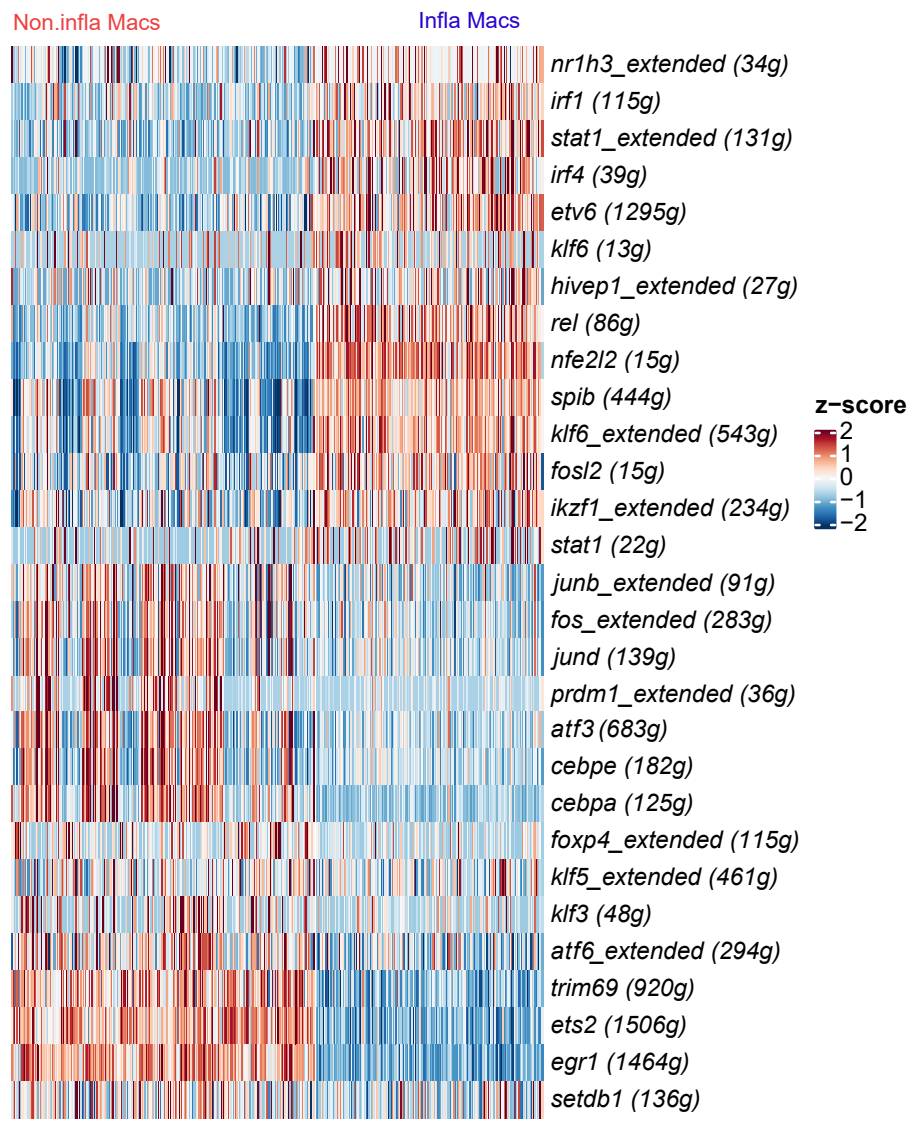

junb_extended (91g) fos_extended (283g)

jund (139g)

prdm1_extended (36g)

atf3 (683g)

cebpe (182g)

cebpa (125g)

foxp4_extended (115g)

klf5_extended (461g)

klf3 (48g)

atf6_extended (294g)

trim69 (920g)

ets2 (1506g)

egr1 (1464g)

setdb1 (136g) 
rịght

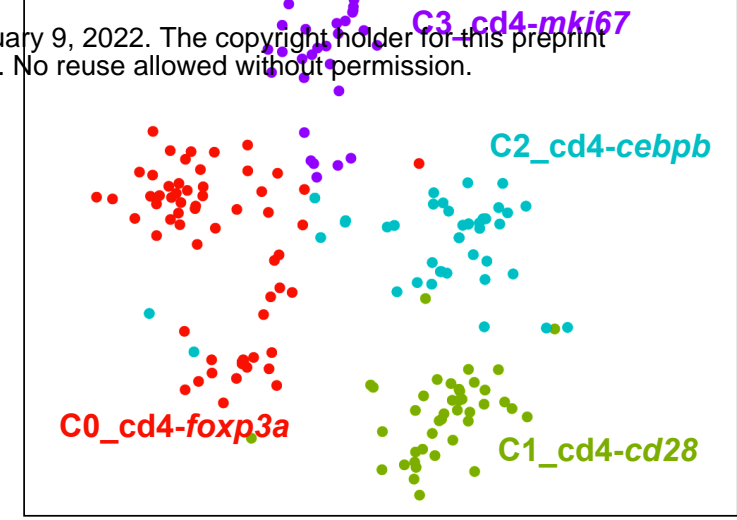

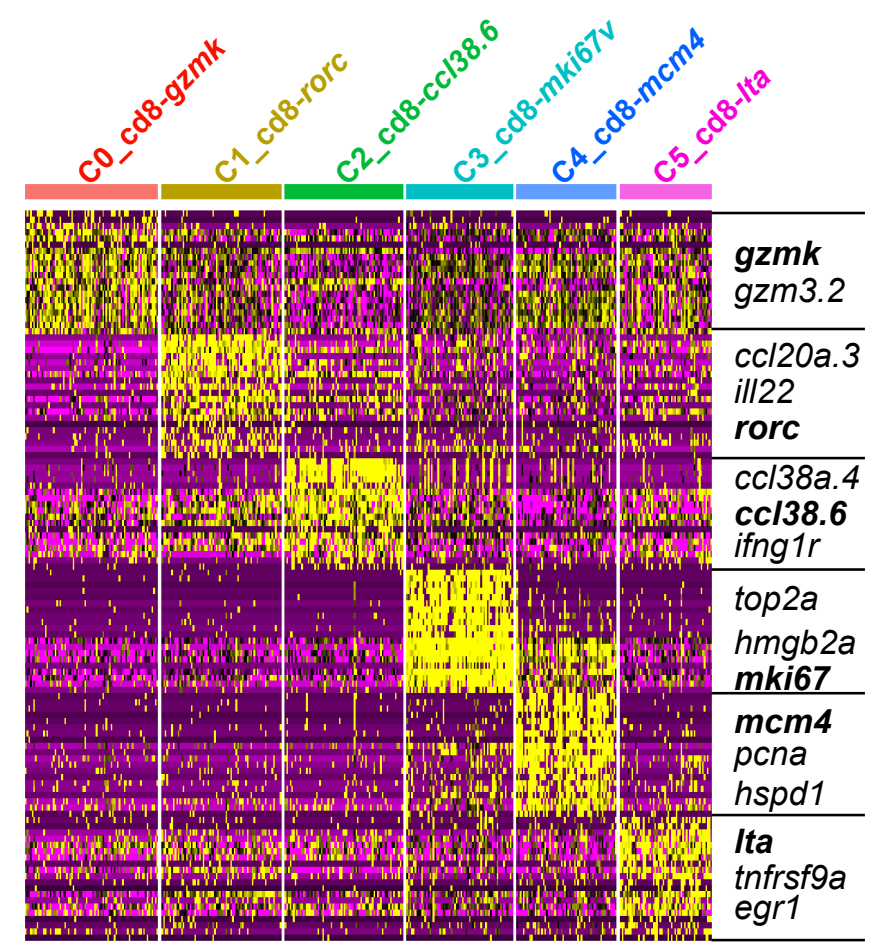

$\begin{array}{cccccc}1.0 & -0.5 & 0.0 & 0.5 & 1.0\end{array}$

Expression

$F$
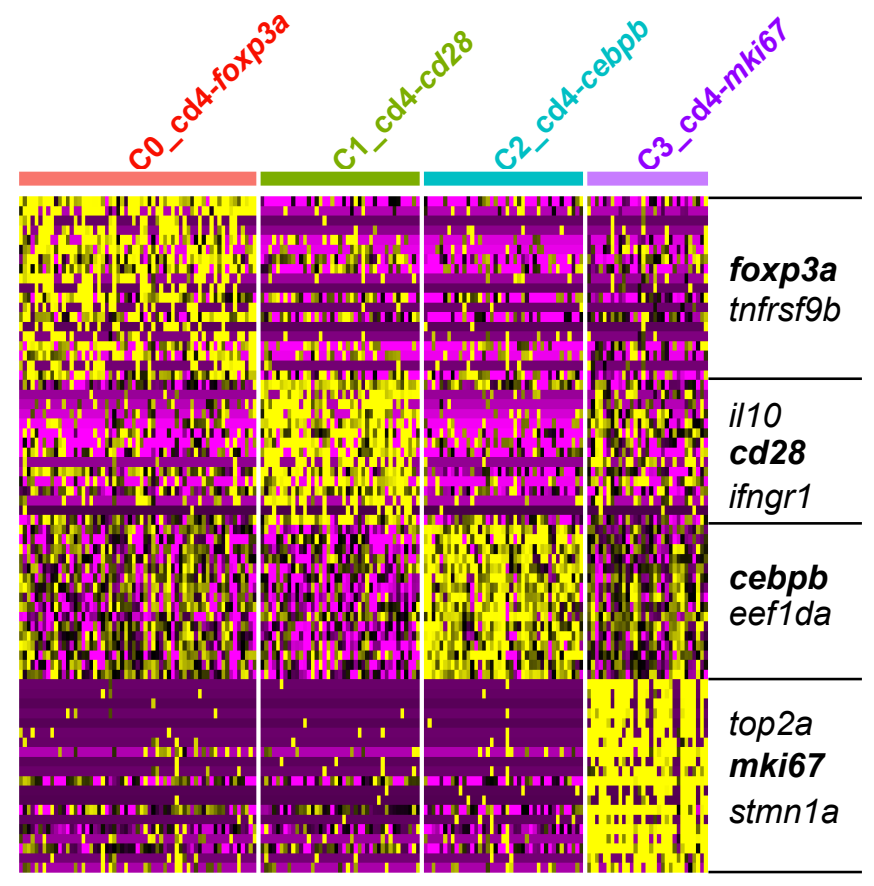

\section{E}

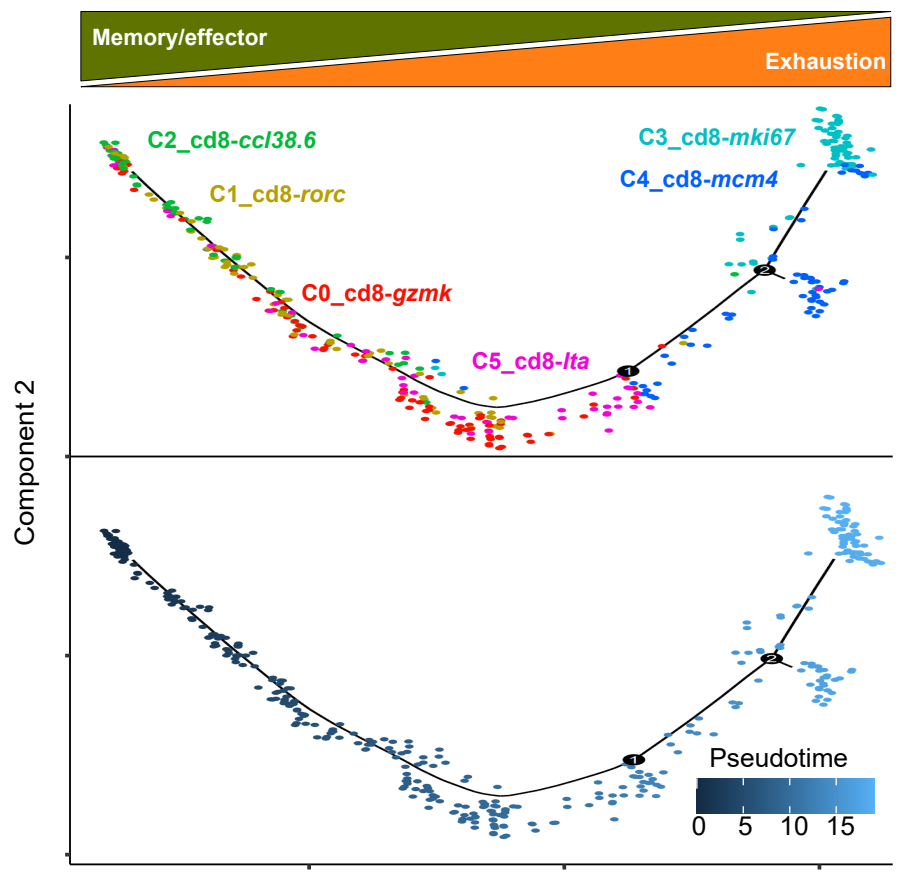

Component 1

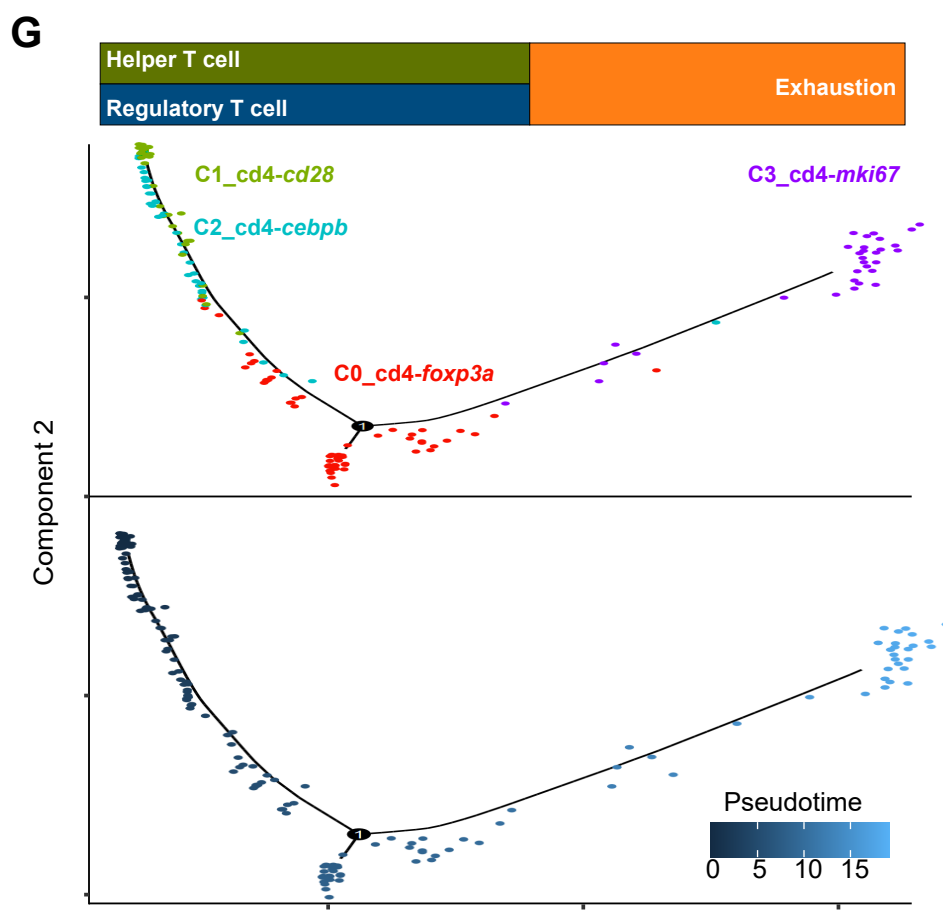


B

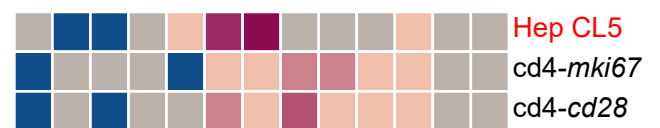

$$
\begin{array}{r}
\text { plxnb2a_ptn } \\
\text { dpp4_cxcl11.6 } \\
1 \text { 1_aLb2 complex } \\
\hdashline
\end{array}
$$

:a:

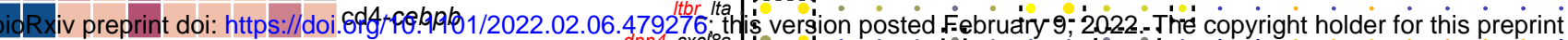

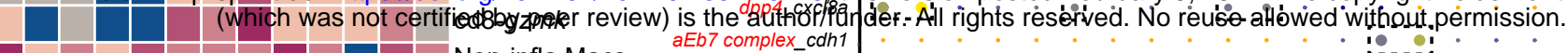

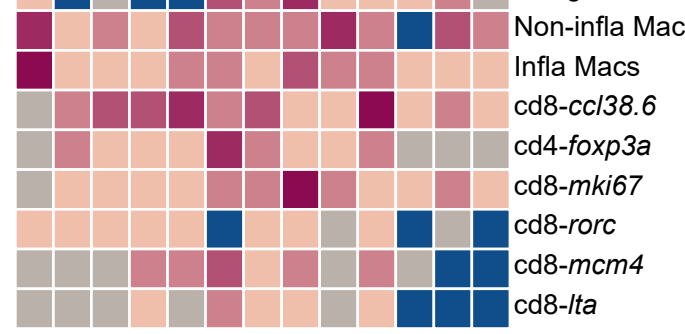

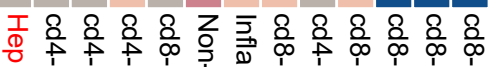

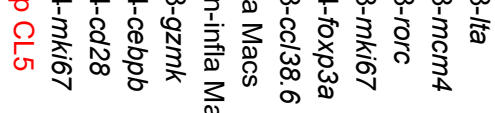

$0.5 \quad 1 \quad 1.5$

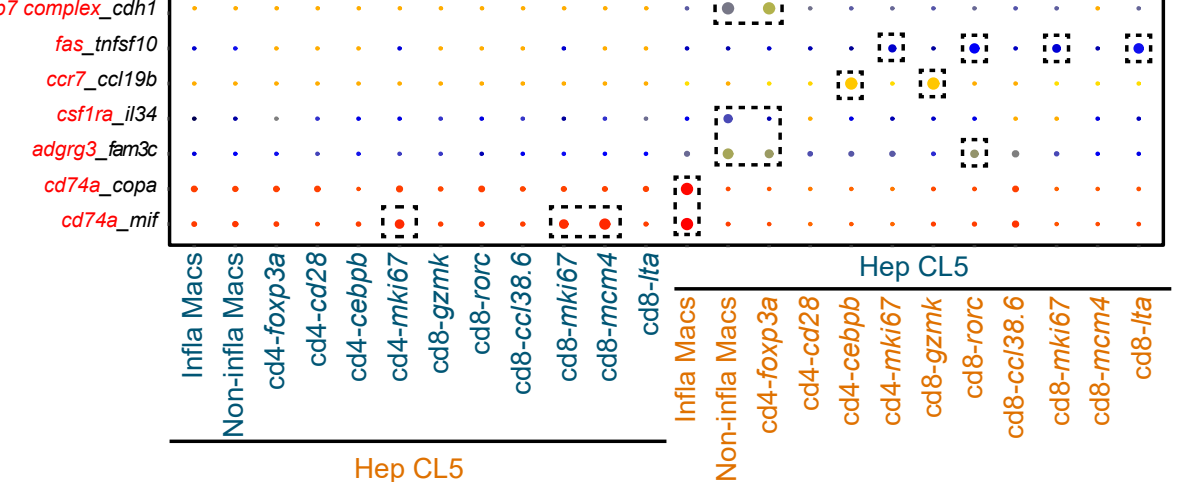

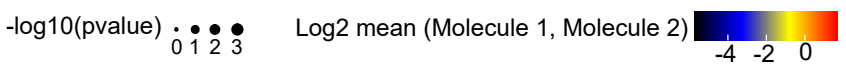

C

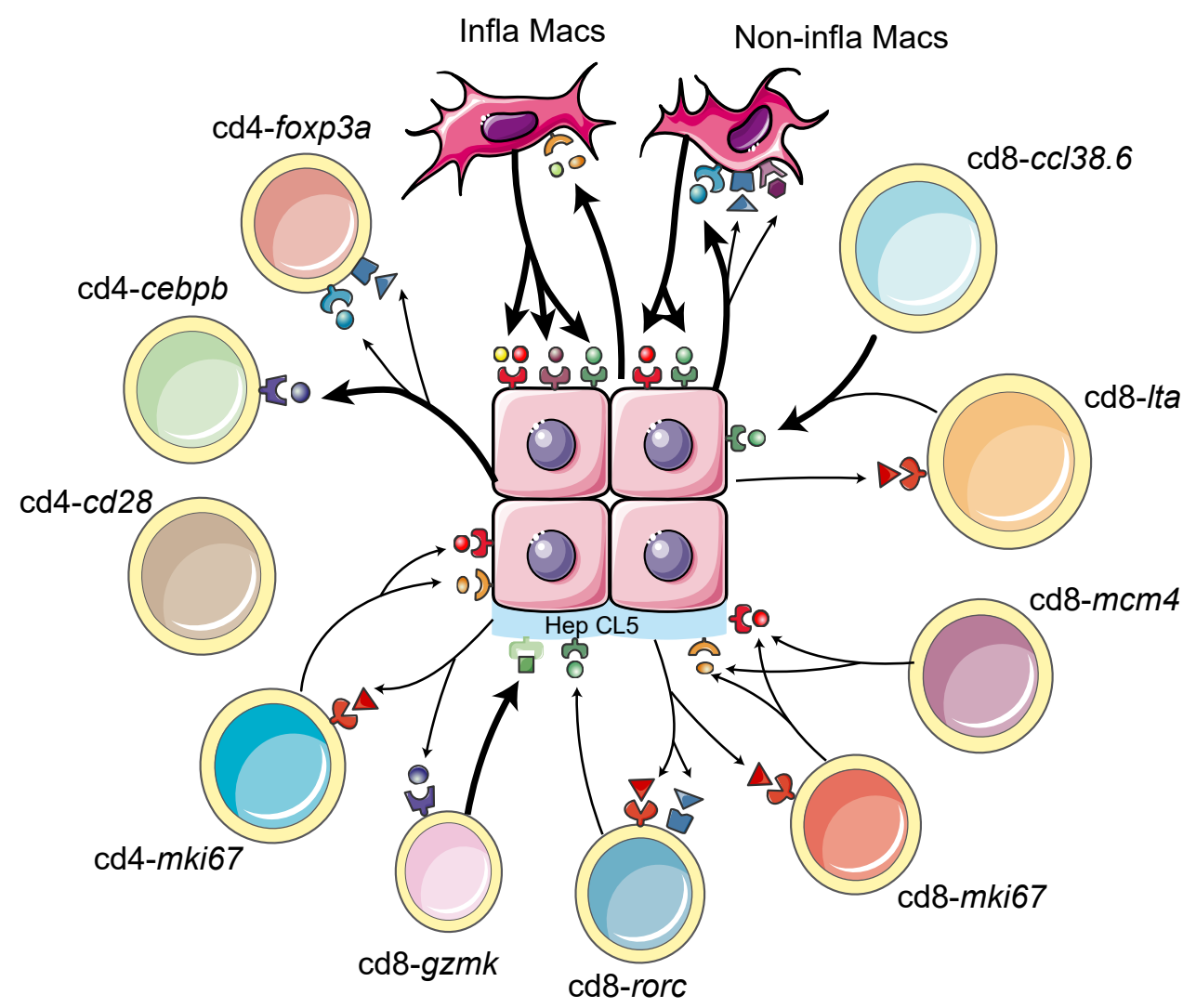

Ligands

Receptors
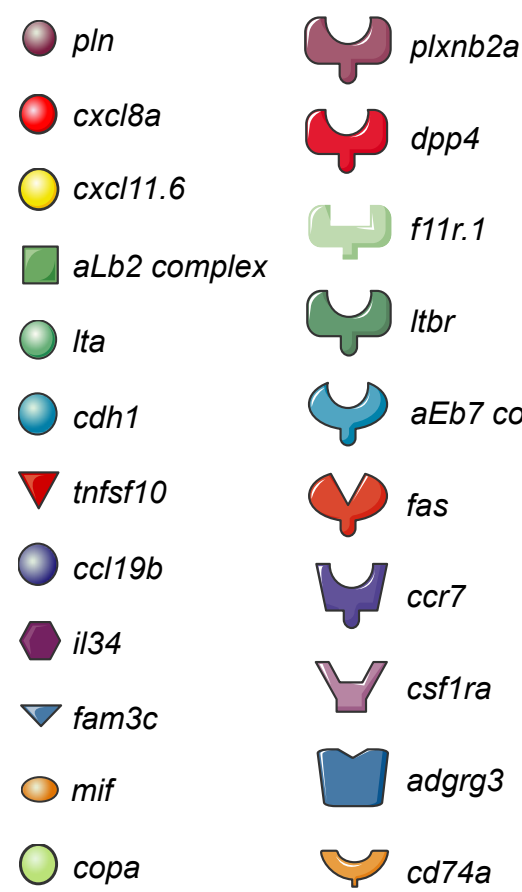

$d p p 4$

f11r. 1

$\bigcup_{1 t b r}$

I aEb7 complex

fas

Ucr7

$\square$ csf1ra

$\square \operatorname{adgrg} 3$

₹cd74a

-log10(pvalue)

$1-2$

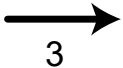

